\title{
Quando il rito inganna: azioni rituali e mechanaŕ in Euripide (El., IT., Hel..*
}

\author{
Enrico Medda \\ Università di Pisa, Italia \\ enrico.medda@unipi.it \\ (iD https://orcid.org/0000-0002-7767-8683 \\ Andrea Taddei \\ Università di Pisa, Italia \\ andrea.taddei@unipi.it \\ (iD https://orcid.org/0000-0002-8977-5528
}

Misleading rites: ritual actions and mechanai in Euripides (El., IT., Hel.)

\section{SOMMARIO:}

Questo lavoro esamina tre casi euripidei nei quali il riferimento ad azioni rituali specifiche (il riconoscimento di un nuovo nato, la celebrazione di un rito funerario, la purificazione di un omicida) funziona come strumento di inganno utilizzato da uno o più personaggi ai danni di un altro. Si tratta di casi in cui il rito assume una precisa funzione drammatica e permette di creare una complicità con l'uditorio, che coinvolge tanto l'autore quanto coloro che, in vari momenti dell'Elettra, dell'Ifgenia tra $i$ Tauri $\mathrm{e}$ dell'Elena, si trovano sulla scena.

PAROLE: Rito, Euripide, inganno, Elettra, Ifigenia fra i Tauri, Elena .

\section{ABSTRACT:}

The article deals with three euripidean passages (from Electra, Iphigenia among the Taurians and Helen) in which the enactment of ritual actions (such as the admission of a newborn child to the familial group, the celebration of a funerary rite, the purification of a murderer) works as a deceiving or misleading resource. In such circumstances rites play a major dramatic role in creating a complicity between the author, the audience and the characters acting on scene.

KEYWORDS: Rite, Euripides, deception, Electra, Iphigenia among the Taurians, Helen .

La relazione tra riti e tragedie può essere affrontata secondo direzioni molteplici e gradi diversi di complessità. Come è noto, nella storia degli studi il problema è stato affrontato da differenti punti di vista, ${ }^{1}$ e ciascuno studioso ha scelto secondo quale ottica inquadrare la questione, a partire dal rapporto controverso e discusso sin dall'antichità- della relazione con Dioniso e con la festa nell'ambito della quale le tragedie erano rappresentate, per arrivare al complesso di valori simbolici rintracciabili dietro il riferimento all'esecuzione (talvolta distorta) di un rito o all'allusione a una specifica festa. Se per il lettore moderno è possibile svolgere analisi di questo tipo è perché quel che noi isoliamo come sfera separata dell'agire sociale, e definiamo azione religiosa, era in realtà parte della vita ordinaria dei $\pi \circ \lambda i \tau \alpha \iota$, che incrociavano la dimensione del rito nel quadro di numerose circostanze come quelle che, per esempio, marcavano il riconoscimento, da parte del gruppo, degli elementi ordinari del ciclo vitale: la nascita, le diverse fasi dello sviluppo fisiologico maschile e femminile, la formazione della famiglia e la complessa gestione dei rapporti all'interno di quest'ultima, il s uperamento della crisi determinata dalla morte dei singoli o di specifiche collettività. Lo stesso può dirsi delle fasi che caratterizzavano l'agire sociale della comunità politica, di volta 
in volta più o meno connesse con il ciclo agricolo, la ritualizzazione dei conflitti e dell'esercizio guerriero, il contenimento delle dinamiche che regolavano il rapporto tra i sessi e i diversi ceti sociali.

Molti dei riti ai quali si è poco sopra fatto -seppure indirettamente- cenno erano organizzati intorno a un ciclo calendariale lunisolare piuttosto complesso e non sempre chiaro per l'interprete moderno, mentre altri numerosi riti entravano in rapporto con i $\pi 0 \lambda i \tau \alpha \iota$ nella dimensione della loro quotidianità, verrebbe da dire dell'ordinarietà del rapporto col tempo e con gli altri. Fare riferimento a queste azioni rituali nel quadro di una performance drammatica significa dunque stimolare la competenza rituale degli spettatori intorno ad elementi che sono parte di un'esperienza che corrisponde alla vita e alle relazioni dei singoli, intesi come parte di una collettività che riconosce a quei riti una forza efficace. Simili riferimenti possono avere uno specifico valore drammaturgico, utile ad arricchire il quadro delle relazioni tra i personaggi attraverso la sollecitazione di un immaginario simbolico che, ad un tempo, struttura e dà forza alla fisionomia di un personaggio e diventa uno strumento nelle mani di questi ultimi, che lo utilizzano per strutturare e, in alcuni casi, dare una svolta agli eventi rappresentati sulla scena.

In questo lavoro intendiamo prendere in considerazione tre casi euripidei nei quali il riferimento ad azioni rituali specifiche (il riconoscimento di un nuovo nato, la celebrazione di riti funerari, la purificazione di un omicida) funziona come strumento di inganno utilizzato da uno o più personaggi ai danni di un altro, quando cioè il rito inganna e permette di creare una complicità con l'uditorio, che coinvolge tanto l'autore quanto coloro che, in vari momenti dell'Elettra, dell'Ifgenia tra i Tauri e dell'Elena, si trovano sulla scena.

\section{ELetTRA ${ }^{2}$}

\section{Vendetta e sacrificio}

Nell'Elettra il nesso fra la vendetta e la pratica sacrificale è particolarmente evidente. Egisto viene ucciso mentre compie un sacrificio alle Ninfe, la cui descrizione è una tra le più dettagliate che si incontrano nel teatro tragico, con menzione dei singoli oggetti coinvolti (canestro, coltello sacrificale, fuoco, lebeti disposti attorno alla $\dot{\sigma} \sigma \chi \dot{\alpha} \rho \alpha$ ) e dei vari momenti del rito (purificazione degli ospiti, gettito di chicchi di grano sull'altare, preghiera, offerta di un ciuffo di peli della vittima, colpo tra le spalle che la abbatte, scuoiamento dell'animale ed esame delle viscere, cf. El. 774-843). In questa sequenza si inserisce Oreste, che si finge viaggiatore tessalo, quando viene invitato da Egisto a dar prova dell'abilità di quel popolo nello scuoiare e fare a pezzi i tori. Oreste chiede un coltello tessalo atto a spaccare il torace, si mette all'opera e, mentre Egisto si china sulla vittima, lo colpisce alla schiena, spezzandogli la spina dorsale. L'usurpatore viene così a coincidere inopinatamente con la vittima sacrificale, e subisce degno contrappasso rispetto alla morte di Agamennone, che già in Eschilo era stata ripetutamente associata all'immagine di un sacrificio corrotto. $^{3}$

Anche la vendetta nei confronti di Clitemestra è caratterizzata dal richiamo a una precisa occasione sacrificale: in questo caso, però, a differenza del sacrificio di Egisto, l'occasione rituale è frutto di finzione, e viene costruita con uno stratagemma da Elettra, che assume un ruolo diretto nella progettazione e attuazione del matricidio. Ai vv. 647-63 la giovane dichiara di avere in mente un modo per attirare la madre presso la sua povera capanna, situata in un'aspra zona montuosa alla periferia del territorio di Argo. Il Vecchio dovrà comunicare alla regina che Elettra ha partorito da dieci giorni, così che la rete in cui l'assassina è destinata a cadere sia preparata a partire da un rito connesso con la finta nuova nascita che verrà annunciata.

\section{I riti della nascita: $\dot{\alpha} \mu \phi ı \rho \rho \dot{\mu} \mu l \alpha$ e $\delta \varepsilon \kappa \dot{\alpha} \tau \eta$}

È necessario innanzitutto chiarire la natura della pratica rituale cui Euripide fa riferimento in questa occasione. È noto che per i Greci antichi il momento della nascita si associava a una impurità della 
donna, che si trasmetteva a chi la aiutava nel parto e più in generale a chi si trovasse sotto lo stesso tetto, protraendosi per alcuni giorni. ${ }^{4}$ Nei primi giorni dopo la nascita del bambino avevano luogo due importanti cerimonie, la cui relazione reciproca non è del tutto chiara: gli $\alpha \mu \phi i \delta \rho o ́ \mu \iota \alpha$ e il sacrificio del decimo giorno $(\delta \varepsilon \kappa \dot{\alpha} \tau \eta)$.

Gli $\alpha \mu \phi ı \delta \rho \dot{\mu} \mu \alpha$ sono per lo più collocati dalle fonti al quinto giorno dopo la nascita, e prevedevano una corsa dei membri della famiglia attorno al focolare con il bambino fra le braccia. I particolari sono incerti, perché per alcuni testimoni (ad esempio lo scolio a Plat. Theaet. 160b) erano le donne a correre, secondo altri invece (Harpocrat. $\alpha 97$ K., Suda $\alpha 1722$ A., Apostol. 2.56) erano gli uomini (nudi secondo Hesych. $\delta$ 2400 L.-C.). Tre fonti (lo scolio sopra citato al Teeteto, la Suda ePaus. Att. $\alpha 101$ Erbse) riferiscono che in quell'occasione le donne che avevano assistito al parto eseguivano prima del rito un lavacro rituale delle mani, recuperando la purezza. ${ }^{5}$ Tutt'altro che unanime è l'interpretazione dell'atto rituale, che secondo alcuni studiosi sanciva la purificazione rispetto alla contaminazione della nascita, secondo altri invece simboleggiava la presa di contatto con il focolare della casa, riconoscendo così l'appartenenza del nuovo nato al $\gamma^{\prime} \varepsilon \dot{v} \circ \varsigma$ del padre. $^{6}$

Per la cerimonia della $\delta \varepsilon \kappa \dot{\alpha} \tau \eta$ le testimonianze più rilevanti ci vengono da Aristofane ed Euripide: essa si teneva al decimo giorno dalla nascita, prevedeva un sacrificio ed era il momento in cui si dava il nome al nuovo nato. ${ }^{7}$ Uno scolio ad Aristoph. Av. $494 \mathrm{e}$ altre fonti lessicografiche riferiscono che in quell'occasione familiari e amici venivano invitati a un banchetto, ${ }^{8}$ che, stando alla testimonianza del comico Eubulo, era seguito da danze che potevano protrarsi per tutta la notte. ${ }^{9}$

$\grave{E}$ certamente a questo secondo rito che fa riferimento Elettra, come risulta dai vv. 1125-26, nei quali chiede

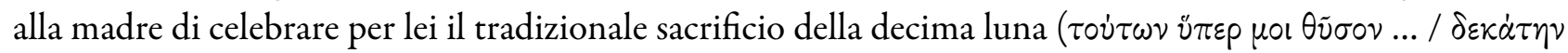

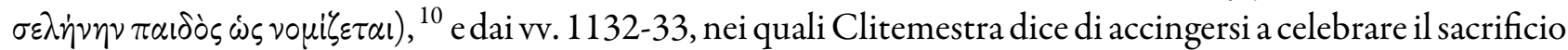

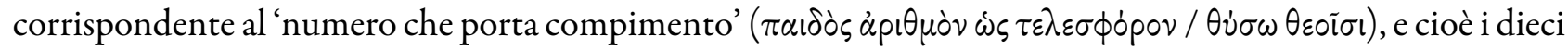
giorni. ${ }^{11} \mathrm{E}$ quasi certo inoltre che il numero dieci sia menzionato anche al v. 654 ( $\delta \dot{\varepsilon} \chi$ ' $\dot{\eta} \lambda$ ious Elmsley: $\lambda \dot{\varepsilon} \gamma^{\prime}$ $\dot{\eta} \lambda$ iovs $L)$, dove Elettra ordina al Vecchio, che le chiede se deve riferire a Clitemestra che ha partorito da pochi

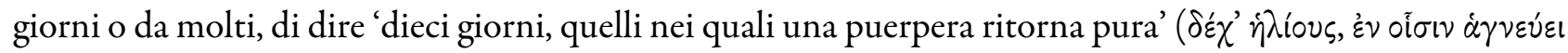
$\lambda \varepsilon \chi(\omega) .{ }^{12}$

\section{Perché proprio quel rito?}

Consideriamo adesso come l'evocazione di questo rito familiare risulti funzionale al progetto drammatico sviluppato da Euripide, decretando il successo del piano d'inganno. Al momento della pianificazione dell'agguato, quando Elettra si dice certa che Clitemestra, sentita la notizia del parto, verrà alla capanna (v.

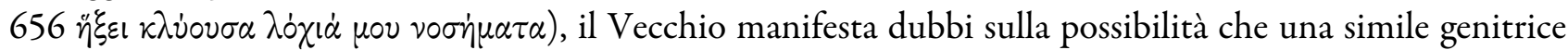

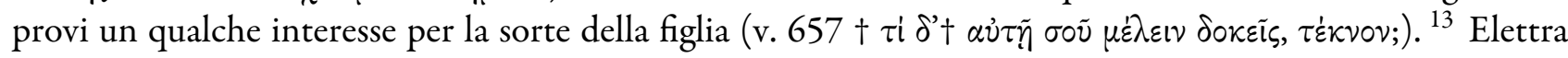
però ribadisce con forza la sua convinzione al v. 658 ( $\nu \alpha$ i, 'si, verrà'). Gli interpreti si sono divisi sulla ragione di tale sicurezza. Secondo M. Kubo, il punto decisivo sarebbe che Elettra chiede di comunicare a Clitemestra la nascita di un figlio maschio (v. 652), dando così corpo alle peggiori paure dell'assassina, che accorre per sincerarsi della situazione e andare poi a riferire a Egisto l'inquietante notizia, con intenzioni perniciose nei confronti del bambino. ${ }^{14}$ Dunque, Elettra conterebbe sulla natura malvagia della madre, facendole pervenire una notizia che non può che sconvolgerla e attirarla nella trappola.

Senonché, dal riassunto della vicenda che il Contadino fa nel prologo della tragedia (vv. 19-42) si ricava che al tempo dell'uccisione di Agamennone Clitemestra aveva manifestato un residuo di amore materno sufficiente a proteggere Elettra dalla furia assassina di Egisto. La soluzione ideata da quest'ultimo era stata allora di darla in sposa a un uomo di bassa condizione sociale, contando sul fatto che un figlio di nascita non nobile non avrebbe mai potuto proporsi come vendicatore di Agamennone. ${ }^{15}$ Elettra stessa, al v. 267, dice che 
Egisto l'ha fatta sposare con il Contadino perché generasse una prole di basso rango ( $\dot{\alpha} \sigma \varepsilon v \nu \tilde{\eta}):$ la procreazione di figli era dunque prevista fin dall'inizio come un fatto non pericoloso. Se non si è realizzata, è stato solo per la scelta rispettosa del marito, che non ha voluto disonorare una moglie di lignaggio molto più alto del suo.

La nascita di questo bambino, dunque, non soltanto non può suscitare paura in Clitemestra, ma, al contrario, rappresenta il pieno compimento del disegno iniziale, in quanto distrugge per sempre lo status sociale della figlia di Agamennone. ${ }^{16}$ La correttezza di questa linea interpretativa è dimostrata dalla

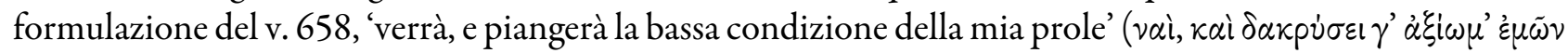
$\tau \dot{\varepsilon} \kappa \nu \omega \nu)$, che riprende con ironia il termine $\dot{\alpha} \xi i \omega \mu \alpha$, che al v. 40 era stato usato per indicare la pericolosa nobiltà

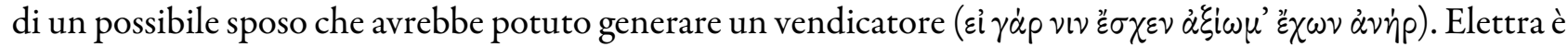
certa dunque che la notizia della nascita risolverà per la madre un problema aperto, e che Clitemestra penserà di avere l'occasione di mostrare la sua compassione ipocrita, assumendo agli occhi del mondo il ruolo della nonna premurosa che assiste la figlia puerpera. ${ }^{17} \mathrm{Ma}$ altrettanto certamente, nella prospettiva di Elettra, per la regina venire a quella casa equivarrà al presentarsi alle porte dell'Ade (v. 662). ${ }^{18}$

\section{Senza vicini, senza amici: l’emarginazione di Elettra}

$\grave{E}$ in questo quadro che si colloca l'ingresso in scena di Clitemestra, caratterizzato da una forte tensione fra la condizione di emarginazione e povertà di Elettra e il lusso della madre, che ha viaggiato su un carro, attorniata da schiave troiane (vv. 998-1003). Elettra intenzionalmente sottolinea la sua condizione quasi servile, e si propone provocatoriamente per sostenere Clitemestra mentre scende dal carro, come farebbe un'ancella. ${ }^{19} \mathrm{Al}$ rifiuto della madre, che le fa notare di avere schiave disponibili per quel compito, la figlia ribatte in modo aggressivo che per lei è naturale comportarsi così, visto che si trova nella stessa condizione delle serve troiane, essendo stata resa orfana e privata della casa paterna (vv. 1004-10).

Le due donne si confrontano in un lungo agone, nel quale Clitemestra dà un'immagine in qualche modo attenuata delle proprie azioni, e si presenta come incline a sopire il contrasto, che viene invece attizzato da Elettra. Ai vv. 1088-90 quest'ultima pone ancora in primo piano il tema delle ricchezze del padre, che sarebbero legittimamente spettate a lei e a Oreste, e che invece la madre ha usato come dote per comprarsi un nuovo marito. Così, quando al v. 1123 Clitemestra cerca di passare a un altro argomento, chiedendo alla figlia perché l'abbia fatta venire, la risposta, che contiene la richiesta di essere lei a compiere il sacrificio del decimo giorno, si connette inestricabilmente con la disastrosa condizione di emarginazione in cui Elettra è precipitata. La finta puerpera si presenta come una giovane inesperta, al primo parto e dunque bisognosa di assistenza; la madre osserva allora, verosimilmente richiamandosi a quella che si può presumere fosse la normalità del rito, che a fare il sacrificio dovrebbe essere la donna che l'ha aiutata a partorire (v. 1128). Ed è qui che scatta il cuore della trappola. Questa donna, secondo il falso resoconto di Elettra, non esiste, perché la sua emarginazione e la sua solitudine sono tali da averla costretta a partorire completamente da sola (v. 1129

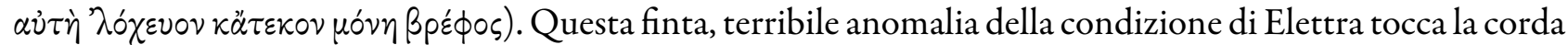
giusta: Clitemestra chiede con meraviglia se la casa sia così isolata da non garantire neppure il supporto di una

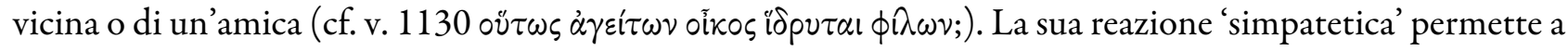
Elettra di portare la stoccata finale, ricordandole che quella condizione è determinata dalla povertà in cui è

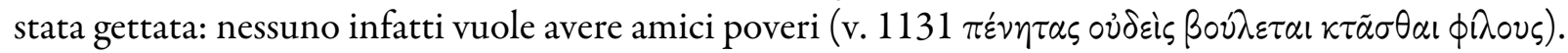

Nel racconto fittizio di Elettra tutti gli elementi che in una situazione di normalità dovrebbero associarsi alla $\delta \varepsilon \kappa \dot{\alpha} \tau \eta$ risultano assenti: nessun banchetto è possibile, non ci sono parenti, né vicini o amici che possano festeggiare la nascita e compiere assieme ai familiari il rito d'uso. Il quadro desolato tracciato dalla figlia fa breccia almeno in parte nell'animo di Clitemestra: la vista di lei, inaccettabilmente sporca e malconcia dopo aver partorito, e incapace di portare a compimento un rito così semplice, la induce ad accettare la richiesta

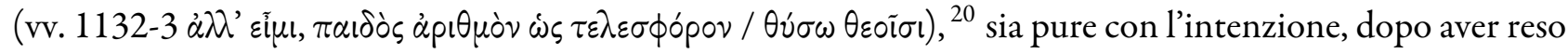


alla figlia quella piccola $\chi \dot{\alpha}$ pıs, di andare subito a raggiungere Egisto nel luogo dove sta sacrificando agli dèi, per rendere anche a lui la $\chi \dot{\alpha} \rho ı \varsigma$ dovuta (vv. 1333-38: si noti in particolare l'uso speculare del termine nel v.

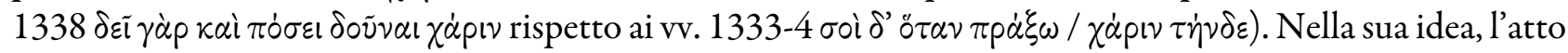
rituale che accetta di compiere le dà la possibilità di apparire, sia pure solo per un momento, come parte della vita di quella casa e di quel nucleo familiare. Ciò di cui non si rende conto è che proprio il desiderio di dare quell'immagine di sé entrando in casa le costerà la vita, a causa dell'ambiguità costruita da Elettra attorno al rito, che comporta un sacrificio di cui la madre stessa sarà la vittima.

\section{Dal rito di nascita al rito di morte}

Mentre entra in casa, Elettra rivolge a Clitemestra una battuta che non può udire, nella quale il piano della realtà affiora in modo spietato: 'Sacrificherai agli dèi la vittima che è necessario sacrificare. È pronto il canestro, e il coltello affilato che ha abbattuto il toro, presso il quale ti abbatterai sotto il colpo: e sarai sposa anche nell'Ade di colui con cui giacevi in vita. Questa charis io ti renderò, e tu a me pagherai la pena per mio padre' (vv. 1141-46). ${ }^{21}$ La morte sacrificale si configura come dura ritorsione contro Clitemestra della $\chi \dot{\alpha}$ pıs ipocrita della quale si era parlato poco sopra, e stravolge il significato dell'atto, che dovrebbe configurare una $\chi \alpha$ ápı nei confronti del divino, deviandola verso l'essere umano assetato di vendetta. La morte di Clitemestra assume così tratti sacrificali strettamente contigui a quella di Egisto, il cui corpo giace nella casa, e con il quale si rinnoverà l'unione amorosa anche nell'Ade (come spesso altrove, Euripide fa ricorso alla pervasiva affinità presente in tragedia fra matrimonio e rito sacrificale). Al tempo stesso, la morte della regina realizza pienamente la dimensione ironica delle parole pronunciate da Elettra al v. 658, quando aveva detto che la madre sarebbe certo venuta per (com) piangere la misera condizione del nipote. In realtà il bambino nato solo nella finzione la fa piangere sulla sua stessa sorte.

La vendetta per un delitto che ha scosso le fondamenta della famiglia degli Atridi si attua in un contesto che sconvolge con altrettanta forza la normalità della vita familiare. Un rito di nascita e di accoglienza si trasforma in rito di morte, grazie alla finzione con cui Elettra disegna il contesto religioso entro il quale intende attuare la sua vendetta. Proprio nel momento in cui tenta di essere almeno per un attimo una madre premurosa, Clitemestra viene punita per aver rinnegato la sua natura e per aver trasformato in un inferno la vita di sua figlia.

\section{IFIGENIA TRA I TAURI ${ }^{22}$}

Che le azioni rituali abbiano rilievo per lo svolgimento dell'azione drammatica nella Ifgenia tra $i$ Tauri è, prima di tutto, una constatazione inevitabile, anche per chi percorra rapidamente lo svolgimento degli eventi nella tragedia. Dai riferimenti iniziali al sacrificio umano cui vengono sottoposti coloro che arrivano presso il santuario di Artemide, attraverso i riferimenti ai Choes ateniesi, ai Delia, alle Brauronie e alle Tauropolie nel finale del dramma, l'allusione più o meno esplicita alle azioni rituali contribuisce a determinare l'articolarsi dell'azione drammatica. ${ }^{23}$

Il riferimento alle azioni sacre sembra talvolta svolgere la funzione di una sorta di chiave, utile alla protagonista del dramma ad avviare ed aprire un canale comunicativo privilegiato con gli spettatori, in grado tanto di fare progredire, e all'occasione di accelerare, gli eventi, quanto di stabilire con il pubblico un'intesa fondata su un comune codice simbolico, all'insaputa di Toante, dei suoi attendenti e, almeno all'inizio, anche di Oreste e Pilade. Con il progredire degli eventi, il riferimento alle azioni sacre diviene infatti un vero e proprio strumento utile alla costruzione del desiderio e, poi, del progetto di fuga verso la Grecia.

La costruzione di un'evasione dalla terra dei Tauri è, in effetti, un tema che percorre il dramma dall'inizio alla fine, da quando Oreste riferisce (vv. 87-92) dell'oracolo di Apollo che vincola il sollievo dalle pene che 
lo tormentano $(\dot{\alpha} \mu \pi v \circ \alpha \dot{\alpha}$... $\pi \dot{v} v \omega \nu)$ al fatto di prendere e portare ad Atene la piccola statua ${ }^{24}$ di Artemide che

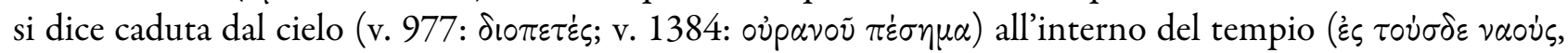
v. 88), fino a quando Atena ex machina darà precise istruzioni sul futuro collocamento a Brauron di quella medesima statua e sull'avvio di una forma innica nuova in onore di Artemide (vv. 1452-57). Si tratta, per il matricida accompagnato dal cugino, di realizzare quello che in un tribunale ateniese sarebbe stato giudicato

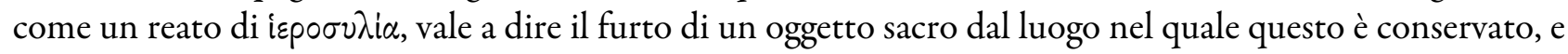
nel quale si suppone esso debba restare. ${ }^{25}$ Come è noto, è grazie all'aiuto della sorella sacerdotessa e custode del tempio, ${ }^{26}$ che il figlio di Agamennone riuscirà nella difficile impresa di sfuggire alla morte che lo attende e nel tentativo di 'trasferire la dea' dalla barbara terra taurica alla prospera e felice Atene. ${ }^{27}$

Accanto alla prescrizione oracolare che vincola Oreste al recupero della statua, bisogna infatti registrare il desiderio di Ifigenia, che in più occasioni appare sospesa tra il rispetto del ruolo nel quale si è trovata a vivere (v. 219: vai $\omega$ ) perché lì è stata collocata (v. $34 \tau i \theta \eta \sigma i \mu \varepsilon)$ da Artemide dopo essere sopravvissuta ai fatti di Aulide, e il rimpianto del proprio destino irrealizzato: Ifigenia ha infatti nostalgia della propria terra e in più occasioni manifesta una presa di distanza dal ruolo che sta svolgendo, insieme ad un desiderio di tornare a casa che troverà concreta realizzazione solo nel finale della tragedia. La fisionomia drammatica di Ifigenia viene dunque costruendosi, sin dall'inizio del dramma, intorno ad un'identità rituale sospesa tra un rigoroso rispetto del ruolo a lei affidato - diciamo così: il suo presente rituale, al quale non è in grado di sottrarsi - e una serie di progressive, crescenti prese di distanza, via via più frequenti e incalzanti. Ed in questo processo di crescente acquisizione di autonomia rispetto alle consuetudini rituali tauriche gioca un ruolo assai importante il riferimento ai riti.

Dopo che Toante ha affidato Oreste e Pilade alla sacerdotessa (v. 335: हैं $\varepsilon \mu \pi \dot{\varepsilon} \sigma \circ 1)$, affinché possa svolgersi il rito sacrificale che comporta l'uccisione degli stranieri ( $\varepsilon \varsigma \chi \varepsilon p \nu 1 \beta \dot{\alpha} \varsigma \tau \varepsilon$ $\kappa \alpha i$ $\sigma \phi \alpha \gamma \varepsilon \tilde{\alpha} \alpha),{ }^{28}$ Ifigenia si dice pronta

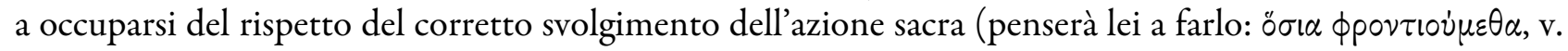
343; è suo dovere pensarci: $\phi \rho \circ \nu \tau \iota \sigma \tau \dot{\varepsilon} \circ v$, v. 468, con una ripresa verbale evidente), dopo avere d'altra parte constatato quanto sia mutato il proprio atteggiamento nei confronti dei Greci. Se, infatti, un tempo ella era

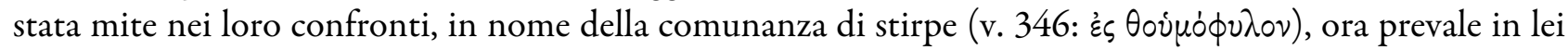
un'ostilità non contenibile, descritta nel discorso pronunciato ai vv. 342-392, che si conclude però anche con una presa di distanza dai $\sigma \circ \phi i \sigma \mu \alpha \tau \alpha$ della dea: Artemide considera impuro chi abbia avuto contatto col

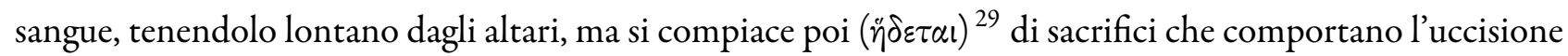

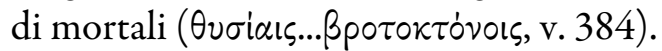

Vengono dunque disegnandosi due linee parallele nell'atteggiamento di Ifigenia rispetto al rito nel quale ella è, suo malgrado, coinvolta. C’è un rito che è inevitabile (v. 620: $\dot{\alpha} \nu \dot{\alpha} \gamma \kappa \eta)$ portare avanti, al quale Ifigenia deve pensare (un aggettivo verbale lo ricorda: $\phi \circ \tau \tau \iota \tau \tau \dot{\varepsilon} \circ v$ ) ${ }^{30}$ e che deve essere svolto con scrupolo (ancora un aggettivo verbale: $\phi \cup \lambda \alpha \kappa \tau \dot{\varepsilon} \circ \nu$, v. 620), ma c'è anche una prima presa di distanza da parte della protagonista, la quale critica l'ambiguità (i $\sigma o \phi i \sigma \mu \alpha \tau \alpha$ di cui si è appena detto) dell'atteggiamento divino nei confronti del sangue, sceglie di occuparsi solo parzialmente del rito sacrificale (lo avvia, ma non lo porta a compimento: v. 40) e condivide con il coro (vv. 384-86) la critica nei confronti di un'azione sacra legittima e, anzi, inevitabile presso la terra dei Tauri, ma inammissibile nella terra d'origine di entrambe.

È come se lo spazio di azione della figlia di Agamennone presso la terra dei Tauri si scavasse una via negli interstizi dell'intreccio complesso tra queste due linee di rispetto e di violazione del rito. Ed è in questi interstizi che Ifigenia costruisce il proprio piano di fuga.

\section{Ingannare persuadendo}

Un vero punto di svolta, concreto ed esplicito, all'interno della linea di rispetto del rito è individuabile nel momento in cui Ifigenia richiama l'attenzione dei suoi interlocutori (e del pubblico) con un invito 


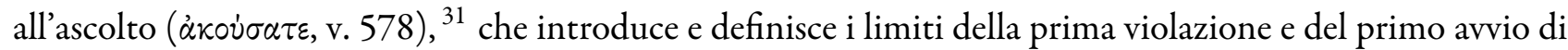
inganno. Con lo scopo di trasmettere un messaggio ad Argo, Ifigenia sceglie infatti di risparmiare uno dei due stranieri (un lungo dialogo tra i prigionieri condurrà all'individuazione di Pilade: vv. 674-715), che sarà a lei vincolato grazie a un lungo rituale di giuramento nel quale il rispetto delle forme risulta addirittura ossessivo. ${ }^{32}$ Ad una prima, seppure parziale, violazione rituale corrisponde dunque immediatamente la costruzione scrupolosa di un rito i cui dettagli sono descritti in modo scrupoloso, quasi esasperato fino al limite -a mio avviso non valicato- dell'ironia. Il filo del rispetto rituale è costruito, d'altra parte, in modo coerente con quanto il pubblico si attende quando è in gioco un sacrificio, e con quanto almeno parte del pubblico può sapere ${ }^{33}$ dei sacrifici umani che si svolgono presso la terra dei Tauri.

Avviando un meccanismo che oscilla, fino al termine della tragedia, tra l'incatenamento e la liberazione delle mani dei prigionieri, Ifigenia impartisce ai suoi aiutanti l'ordine di sciogliere le mani degli stranieri (v. 469) ${ }^{34}$ in ragione del fatto che -come accade per gli animali che vengono sottoposti al sacrificio- ${ }^{35}$ essi sono ora pronti per essere consacrati (iєpoi). A coloro che l'aiutano presso il tempio Ifigenia dà, inoltre, l'indicazione di entrare nel tempio e di preparare $(\varepsilon \dot{\jmath} \tau \rho \varepsilon \pi i \zeta \varepsilon \tau \varepsilon)^{36}$ tutto ciò che serve per lo svolgimento di un rito ancora non qualificato esplicitamente come sacrificio umano, come invece accadrà più avanti, quando proprio il verbo $\varepsilon \dot{\tau} \tau \rho \varepsilon \pi i \zeta \omega$ sarà ripreso in forma leggermente variata ( $\pi \alpha \rho \varepsilon v \tau \rho \varepsilon \pi i \zeta \omega$, v. 725$)$.

Un notevole grado di dettaglio viene mantenuto anche quando si tratta di descrivere il sacrificio umano che attende Oreste. La fitta serie di domande che il fratello rivolge alla sorella durante la sticomitia dei vv. 617-26 contribuisce infatti a costruire, nelle parole dei personaggi e quindi nell'immaginario degli spettatori, un quadro assai preciso e dettagliato di ciò che attende i prigionieri una volta arrivati nella terra dei Tauri.

Desiderando capire di quale morte dovrà morire, Oreste pone una serie di questioni importanti, prima di tutto perché si tratta di distinguere chi avrà la funzione di svolgere il rito sacrificale nel suo complesso (v. 617: $\theta \dot{v} \sigma \varepsilon 1 ~ \delta \grave{\varepsilon} \tau i \varsigma){ }^{37}$ da chi materialmente sgozzerà la vittima (v. 623: $\left.\sigma \phi \alpha \gamma \varepsilon \dot{\varsigma} \varsigma\right)$. Si tratta di una precisazione non banale, utile a sollecitare un atteggiamento di sorpresa da parte del pubblico (non è funzione femminile,

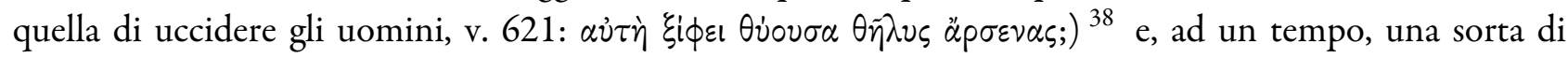
rassicurazione: altro è infatti il ruolo di Ifigenia nei confronti della dea (v. 618), pure descritto come compito tutt' altro che invidiabile (v. 619), altra è la funzione di chi concretamente si occuperà dell'uccisione, che dovrà tra l'altro svolgersi all'interno del tempio (v. 624: है $\sigma \omega \delta \delta^{\prime} \mu \omega \nu \tau \tilde{\omega} \nu \delta \delta^{\prime}$, v. 626: है $v \delta \circ \nu$ ), un luogo che viene via via connotandosi -anche grazie alle scelte verbali di cui si è appena detto- come sede di preparazione delle azioni sacre che poi saranno vero e proprio motore per la progressione degli eventi. ${ }^{39}$ È sulla soglia del tempio che Toante viene fermato (v. 1159) da Ifigenia, che da lì orchestra la costruzione di un inganno rituale fondato sulla persuasione. ${ }^{40}$

Tratteniamo allora anche questi dati e riprendiamo il filo del nostro ragionamento seguendo un percorso che si muove sul doppio binario -in un certo senso ramificato- di cui si è detto. Valutando, non se fuggire, ma quale via di uscita (v. 875: $\tau i v \alpha$ Tópov) ${ }^{41}$ costruire per se stessa ed il fratello, Ifigenia cerca infatti di rispettare l'oracolo di Apollo che, da una parte, impone e legittima la sottrazione e lo spostamento della 'statua caduta dal cielo' (vv. 977-78: $\left.\delta 10 \pi \varepsilon \tau \dot{\varepsilon} \varsigma_{. . . .} \alpha \gamma \alpha \lambda \mu \alpha\right)$ e, dall'altro, permette alla protagonista di evitare di uccidere il fratello

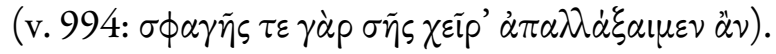

Unici ostacoli alla realizzazione del piano sono lo scrupolo nei confronti della dea (vv. 995-96) ${ }^{42}$ e il timore di una punizione capitale da parte di Toante, quando il sovrano scoprirà che la statua è stata spostata dal suo basamento. Non è tuttavia l'espressione di timore ( $\delta \dot{\varepsilon} \delta \circ \iota k \alpha)$ ad essere per noi rilevante, quanto piuttosto il fatto che per Ifigenia si tratta di escogitare un espediente fondato sul ragionamento e sulle parole

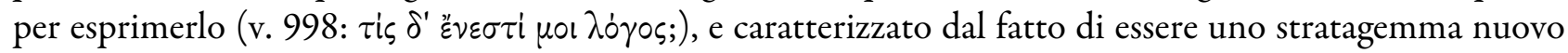

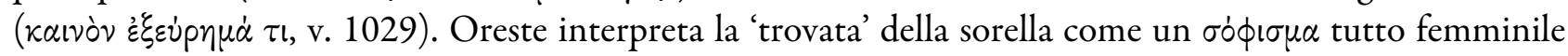
(vv. 1031-32), ma Ifigenia rivendica il percorso che ella inizia a costruire come una scelta, da lei preferita a 
soluzioni più sbrigative, come quella di uccidere il sovrano, un'ipotesi che violerebbe una diversa dimensione

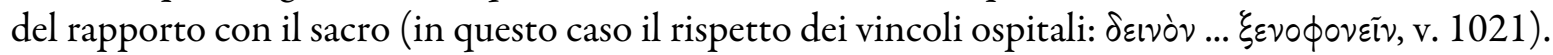

La figlia di Agamennone vuole dunque convincere, persuadere e, per questa via, ingannare Toante, e non eliminarlo. Dall'interno del tempio, dopo avere bloccato il sovrano nello spazio antistante l'ingresso (v. 1159:

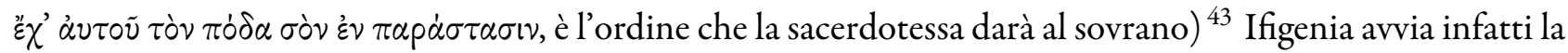

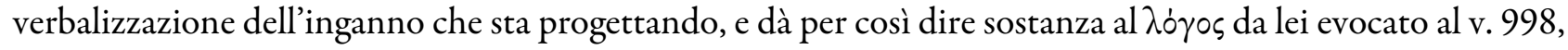
oscillando tra la necessità di rispettare la norma stabilita ( $\theta \dot{\varepsilon} \mu \iota s:$ v. 1035) e l'opportunità di costruire, sopra questa linea di rispetto, piccole deviazioni utili a realizzare il piano da lei escogitato.

La progressiva costruzione dell'inganno viene dichiarata ruotando intorno a verbi che hanno rapporto con la sfera della parola. Secondo una cadenza quasi ritmata, ad ogni intervento nella sticomitia Ifigenia userà un futuro utile a costruire, a vantaggio di Oreste e del pubblico, l'immagine dell'inganno: ella dirà pubblicamente che Oreste è un omicida (o meglio, proclamerà Oreste omicida: $\phi \circ v \varepsilon \dot{\varepsilon} \alpha \sigma \varepsilon \phi \dot{\eta} \sigma \omega$, v. 1033) costruendo

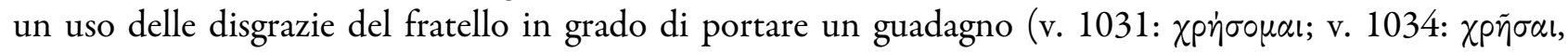

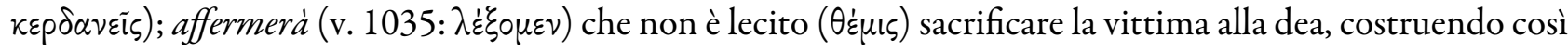
un'opposizione tra lo stato di contaminazione legato all'omicida e il rispetto, da parte degli uomini, del sacro

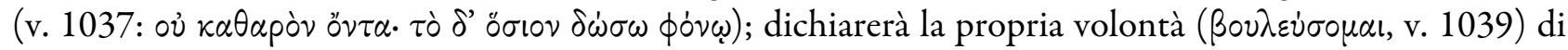
purificare l'omicida in mare; e infine dirà ( $\dot{\rho} \rho \tilde{\omega}, \mathrm{v} .1041)^{44}$ che è necessario lavare in mare anche la statua, che Oreste teme concretamente -esprimendo una constatazione che sembra una provocazione- di non riuscire a

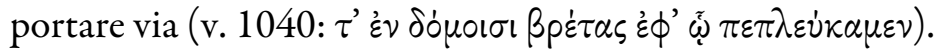

Accanto alla deviazione rispetto alla norma rituale, che è comunque necessario rispettare nelle sue linee

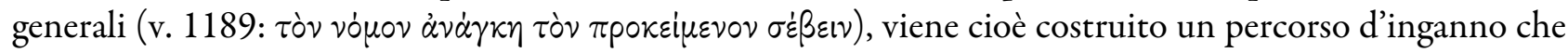
deve risultare plausibile per il sovrano dei Tauri. I dubbi e lo scetticismo espressi da Oreste subito dopo le parole della sorella servono a corroborare l'immagine del rito non ancora eseguito ma, per così dire, fabbricato passo passo davanti agli occhi degli spettatori, con il riferimento alla necessità di purificare lavandola in mare (v. 1039: $\dot{\alpha} \gamma \nu \tilde{i} \sigma \alpha \iota$, v. 1041: vi $\psi \varepsilon \iota v)$ la statua ${ }^{45}$ della dea, in ragione del fatto che Oreste l'ha toccata ( $\sigma \circ \tilde{v}$

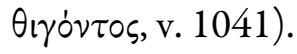

Via via che assume su di sé il controllo progressivo dello svolgimento dei futuri eventi, insomma, Ifigenia costruisce, con il pubblico, un'intesa fondata sulla comune condivisione delle pratiche rituali che trovano appigli in singoli momenti di una specifica festa, senza per questo alludere alla festa in questione nella sua totalità. Mi riferisco alla purificazione in mare ${ }^{46}$ (un passaggio importante nella preparazione dei Misteri eleusini) e al lavaggio degli ornamenti - e più in particolare delle vesti - della dea, praticato in occasione dei Plynteria ateniesi. ${ }^{47}$ Non pare cioè essere in gioco qui, come accade altrove in Euripide, il riferimento al complesso simbolico di una $\dot{\varnothing} \circ \tau \tau \dot{\eta}$, ma sembra coinvolta piuttosto la sollecitazione della competenza rituale del pubblico, che riconosce e per così dire legittima insieme ad Oreste la credibilità e la validità del piano progettato dalla protagonista.

Dopo averla enunciata al v. 743, e ripresa al v. 1020, Ifigenia ribadisce subito dopo la propria scelta

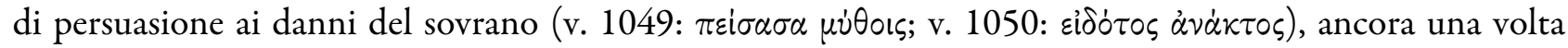
caratterizzata come opzione consapevole rispetto ad una ipotesi diversa, in questo caso quella di agire a sua insaputa $\left(\lambda \dot{\alpha} \theta \rho \alpha\right.$, nelle parole di Oreste al v. 1048). ${ }^{48} \mathrm{Si}$ tratta di una via fondata sulla tessitura di una rete di

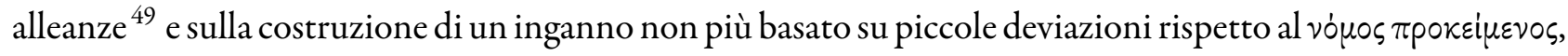
ma ora programmato nei dettagli (cosi accade nell'intero terzo episodio: vv. 1057-1233) e poi reso effettivo, concreto e, per così dire, afferrabile agli occhi degli spettatori grazie al discorso che la protagonista pronuncia al termine del dialogo con il fratello, e prima dello stasimo.

Si tratta di un discorso articolato in tre parti ciascuna di quattro versi, tutte significative: c'è la descrizione dell'uscita dal tempio di Oreste e Pilade accompagnati da tutto ciò che è necessario per il sacrificio (vv. 1222-25); c’è la proclamazione affinché tutti i $\pi$ o $\lambda \tilde{i} \tau \alpha \iota$ taurici restino al chiuso e stiano in disparte (vv. 
1226-29); e c'è, infine, una delle preghiere ${ }^{50}$ che nel corso del dramma Ifigenia rivolge alla 'sua' dea, fatta di una richiesta di ritorno in patria e della promessa, per la divinità, di poter abitare finalmente in un luogo puro, $k \alpha \theta \alpha$ ó̀ (v. 1231).

\section{L'inganno, finalmente}

La progressiva "crescita drammaturgica" di Ifigenia conosce un ulteriore e importante momento di passaggio durante il lungo dialogo con Toante, quello in cui il progetto di inganno conosce una sorta di prima attuazione virtuale: la trappola viene cioè verbalmente costruita a danno del sovrano taurico, ma l'informazione relativa alla concreta messa in opera viene ancora differita, e sarà necessaria la ṕñ Messaggero ai vv. 1327-1419 per averne una descrizione concreta.

Nella tessitura dell'inganno si determina una sorta di riconfigurazione dei rapporti di forza tra i personaggi, tanto nell'accrescimento del peso specifico di Ifigenia, che ha già fatto valere la sua autorità sacerdotale anche sul fratello, ${ }^{51}$ quanto nella strutturazione del personaggio di Toante, re barbaro tra i barbari, che intende rispettare il culto (vv. 996-99) controllandone lo svolgimento (vv. 1080-81), e che ha affidato quest'ultimo a una sacerdotessa (vv. 334-35), alla quale egli a più riprese ribadisce la propria fiducia incondizionata. ${ }^{52}$

La narrazione progettuale costruita da Ifigenia è basata su una verità ben costruita (Oreste è oggettivamente contaminato) e, per così dire, adagiata su un fondo rituale il cui funzionamento deve essere rispettato ( $\dot{\alpha} \dot{\alpha} \gamma \kappa \eta$, v. 1189; $\delta \varepsilon \tilde{\imath}$, v. 1197; $\dot{\alpha} \gamma \nu 1 \sigma \tau \dot{\varepsilon} \circ \nu$, v. 1199): esso viene in effetti rispettato, pure attraverso un uso sapiente di piccole deviazioni che consentono ai tre personaggi di giungere fino alla fuga. Un simile meccanismo può funzionare solo se il pubblico -sollecitato nella sua competenza rituale- riconosce come efficaci le azioni sacre usate come strumento di persuasione ai danni di Toante.

Pare a me, tuttavia, uno sforzo vano quello di cercare coerenza reciproca tra le diverse azioni sacre menzionate. È senz'altro più che probabile, per esempio, un'allusione ai Plynteria (v. 1192), con una ripresa del v. 1041 e alla purificazione in mare che precede i Misteri di Eleusi, ma né il lavaggio della veste di Atena né l'iniziazione misterica sembrano un referente concreto, un quadro all'interno del quale l'azione scenica viene inserita. Tanto meno, mi pare necessario attribuire una funzione per così dire metapoietica ${ }^{53}$ alle parole di Ifigenia, che realizzerebbe un'illusione di primo livello (la finzione del rito) seguita da un'ulteriore illusione di secondo livello (la mancata attuazione del medesimo) utile a trasformare la cancellazione del $\mu$ i $\alpha \sigma \alpha \alpha$ nella maturazione, in Oreste, di una questione di coscienza interiore poi recepita e rappresentata in altri drammi euripidei.

L'elenco degli oggetti che la sorella di Oreste vede uscire dal tempio insieme agli stranieri allude d'altra parte ad una serie di elementi che corrisponde a un complesso non necessariamente coerente, ma senz'altro almeno credibile, ${ }^{54}$ connesso con una purificazione che prevede il sacrificio di agnelli e un uso delle fiaccole, che richiama da un lato l'esperienza eleusinia ${ }^{55}$ e dall'altro contribuisce a legittimare, al v. 216 , la correzione di Reiske $\pi \nu \rho \sigma \tilde{\omega}$ al posto di $\chi \rho v \sigma \tilde{\omega}$ di L, una lezione in sé peraltro non implausibile da un punto di vista storico-religioso. ${ }^{56}$

Nel lungo dialogo tra Ifigenia e Toante si affiancano elementi 'tecnici' del sacrificio e allusioni ad azioni la cui pertinenza rituale è nulla, ma che concorrono a riequilibrare i rapporti di forza a favore di Ifigenia: quando quest'ultima sputa per esprimere il proprio disprezzo, coinvolge Toante nella responsabilità della scelta delle vittime, quasi rimproverando lui e i Tauri per averle trovato prede sacrificali contaminate (vv. 1161-63). Il sovrano va piano piano trasformandosi da interlocutore ad aiutante, e svolge la sua funzione in modo zelante, domandando ad Ifigenia se gli strumenti per il rito siano o meno pronti (v. 1190), chiedendo

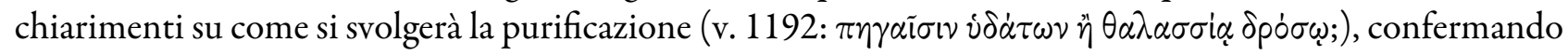

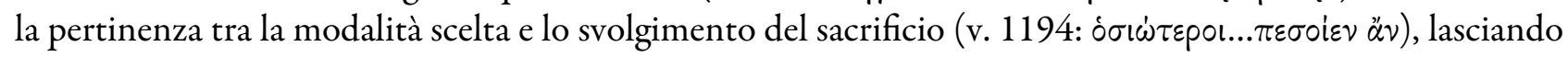

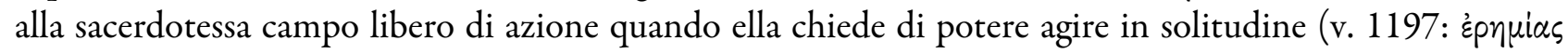




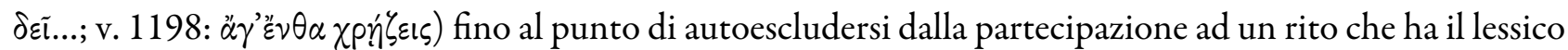

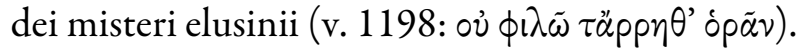

Questa collaborazione si realizza con la crescente approvazione da parte di Toante, che loda l'astuzia di Ifigenia (v. 1180), il suo rispetto della dea (1186), l'adesione alla 'causa' taurica, fino al comune -ma oppostoaugurio che il rito riesca bene, un auspicio espresso in un solo verso (v. 1220) che è un capolavoro di ambiguità, perché Toante risponde con $\sigma v v \varepsilon \dot{\chi} \chi \rho \mu \alpha \iota$ quando Ifigenia si augura che il $\kappa \alpha \theta \alpha p \mu o ́ s$ vada come lei desidera ( $\varepsilon i$

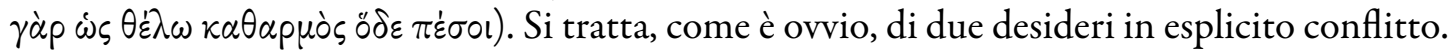

Il carattere necessario dello svolgimento del rito è ora, dunque, orientato nella direzione del rituale simulato. Se all'inizio del dramma Ifigenia subiva la necessità del rito, ed era obbligata a svolgere almeno la parte iniziale del sacrificio umano (anche quando sceglie di salvare uno dei due stranieri, ella dà per acquisito di dovere sacrificare l'altro), ora questa medesima necessità viene utilizzata per convincere un sovrano che va trasformandosi in una sorta di aiutante. Giocando ancora sulla mobilità tra spazi esterni ed interni, dopo avere sfruttato a proprio favore la fiducia di Toante, Ifigenia riesce nella sua opera di persuasione, tanto che è lo stesso sovrano ad inserire nell'argomentazione costruita dalla sacerdotessa degli elementi che addirittura anticipano il raggiro a proprio danno: così accade per esempio quando è lo stesso sovrano a suggerire il fatto che la statua sia portata fuori dal tempio proprio per la purificazione (v. 1176: $\tau \tilde{\omega} \nu \delta^{\prime}$ हैk $\left.k \tau \iota\right)$, una ragione esplicitata da Ifigenia con argomenti che sono in linea con il trattamento giudiziario dei casi di omicidio, che dovevano svolgersi all'aperto. ${ }^{57}$

Il processo di persuasione è riuscito a tal punto, che il sovrano manifesta una sorta di cecità di fronte all'evidenza. Toante pone infatti domande pertinenti sullo svolgimento del rito, ${ }^{58}$ e chiede a Ifigenia di parlare chiaro ( $\dot{\xi} \xi \alpha \dot{\delta} \delta \alpha \sigma \alpha \phi \tilde{\omega} \varsigma$, v. 1162, che riprende il $\sigma \alpha \phi \tilde{\omega} \varsigma$ usato dal Coro al v. 1156). Quando tuttavia egli si avvicina alla verità (v. 1182-84), essendo in questo involontariamente guidato dalla stessa Ifigenia, finisce per anticipare egli stesso l'inganno della sua interlocutrice, dando per acquisito ( parte della dea e quindi dei Tauri. La sacerdotessa ha così buon gioco nel riprendere il filo del suo inganno e ribadire il proprio odio nei confronti dei Greci (v. 1187), una tema già anticipato sin dal v. 346.

Lo scambio di posizione tra la sacerdotessa e il sovrano (al v. 1222 la prima guida la $\pi \circ \mu \pi \dot{\eta}$ verso il mare, mentre il secondo rientra) marca infine la svolta definitiva per la realizzazione della trappola, e questa volta si tratta della realizzazione reale e concreta.

Sembra quasi di potere affermare, infatti, che la ṕñ idealmente le porte del tempio, e rivelare finalmente l'inganno che in quel luogo è stato a lungo concepito, preparato e tenuto nascosto, e che ora può svolgersi (anzi, si è già svolto) nel luogo solitario richiesto da Ifigenia, e concesso senza alcun problema da Toante.

Entrando in scena, il Messaggero si rivolge ai $\nu \alpha \circ \phi \dot{\lambda} \alpha \kappa \varepsilon \varsigma$ che si trovano dentro il tempio (il dato è ribadito a più riprese: vv. 1286, 1287, 1304) fermandosi sulla soglia della porta (v. 1305: '̇े $\pi \dot{v} \lambda$ als), dove Toante era stato bloccato da Ifigenia e dove il sovrano tornerà al v. 1307, quando esce per rispondere all' $\not \partial \gamma \varepsilon \lambda ం \varsigma$.

Di fatto, la descrizione dello svolgimento del rito non è altro che la presa d'atto di tutto ciò che sinora è stato pazientemente costruito da Euripide: l'inganno è perfettamente riuscito e i sacrifici di purificazione vengono

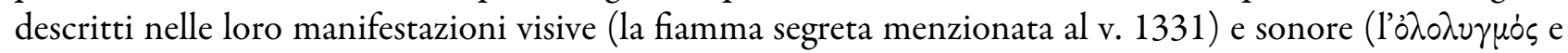
i $\beta \dot{\alpha} \rho \beta \alpha \rho \alpha \mu \dot{\varepsilon} \lambda \eta$ dei vv. 1337-38), narrati da un messaggero che insiste su quanto evidentemente sospetto (vं $\pi 0 \pi \tau \alpha \mu \dot{\varepsilon} v$, v. 1334) fosse quel rituale per chi avesse occhi per vedere. Gli schiavi hanno sì rispettato la

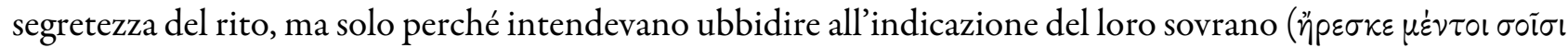
$\pi p \circ \sigma \pi \dot{\lambda} \lambda \circ\left\llcorner, \alpha^{2} \nu \alpha \xi\right.$, v. 1335), il quale finisce così per dare -quasi post eventum- un ulteriore contributo a un inganno evidentemente intuito come tale dagli stessi servi. Sono questi ultimi a rompere gli indugi e, finalmente ( $\tau \dot{\varepsilon} \lambda \circ \varsigma \varsigma \dot{\varepsilon}$, v. 1343), a prendere il coraggio di violare il rito così a lungo tenuto segreto, recandosi

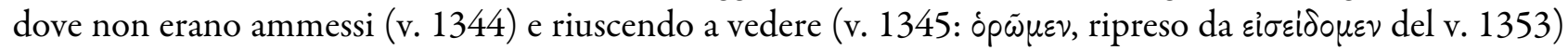

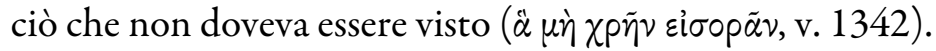


La constatazione è semplice, ed è riassumibile nella formulazione usata dal messaggero per descrivere ciò che Ifigenia, Oreste e Pilade avevano posto in atto, nel segreto del luogo cercato e del rituale progettato, così a lungo descritto e discusso: si trattava infatti di $\delta \dot{\partial} \lambda \iota \alpha \tau \varepsilon \chi \nu \eta \dot{\mu} \mu \alpha \tau \alpha$ (v. 1355), corrispondenti ai $\delta \dot{\partial} \lambda \iota \alpha \kappa \alpha \theta \dot{\alpha} p \mu \alpha \tau \alpha$ annunciati dal Messaggero in apertura del proprio discorso.

Erano solo un inganno, dunque, quelle purificazioni, come anche il pubblico sa bene e come invece non è riuscito a comprendere, quasi fino alla fine, Toante.

ELENA 59

Nel novero dei molti elementi di analogia esistenti ${ }^{60}$ tra Ifigenia fra $i$ Tauri ed Elena può essere senz'altro considerato il modo in cui vengono ingannati Toante e Teoclimeno, entrambi raggirati da un personaggio femminile che organizza la fuga dalla terra lontana verso la Grecia.

Come è noto, Menelao arriva in una terra non meno barbara di quella taurica (v. 864: '̇s $\beta \dot{\alpha} \rho \beta \alpha \rho \rho^{\prime} \dot{\varepsilon} \lambda \theta \dot{\omega} \nu$ ) dove -come accade ad Oreste nella Iffgenia - egli rischia di andare incontro ad un destino di morte descritto dalla protagonista usando un lessico sacrificale ( $\dot{\varepsilon} \nu \theta \dot{\alpha} \delta^{\prime} \tilde{\eta} \lambda \theta \varepsilon \varsigma \dot{\varepsilon} \varsigma \sigma \phi \alpha \gamma \dot{\alpha} \varsigma$, v. 778) anch'esso analogo a quello usato nel dramma taurico. ${ }^{61}$ Elena non può che suggerire a Menelao di fuggire al più presto dalla terra in cui è giunto, usando parole che ritornano identiche a loro stesse anche nelle Fenicie, quasi esistesse una sorta di

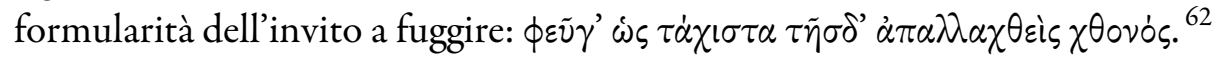

Anche nell'Elena il riferimento alle azioni cultuali gioca un ruolo importante nella tessitura di un inganno che proveremo a osservare da vicino, prestando particolare attenzione agli elementi rituali e al ruolo che questi ultimi rivestono nella costruzione del piano di fuga della coppia di coniugi da poco ricongiunta, dopo che ha mutato il suo corso un destino che Elena e Menelao desiderano volgere a loro favore (vv. 644-46).

In linea generale, un primo elemento da osservare riguarda la strategia seguita dai personaggi, perché anche nell' Elena il fatto di affidarsi a un inganno fondato sul rito è il frutto di una precisa opzione, preferita a quella che sarebbe passata per l'uso della forza, una via teoricamente più conforme allo statuto eroico dell'Atride da poco arrivato in Egitto.

A Menelao che le suggerisce vie più sbrigative Elena spiega infatti che la strada di uccidere il sovrano locale

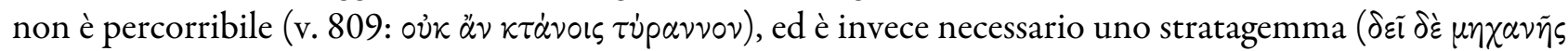

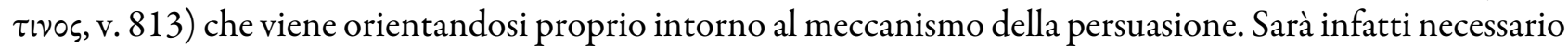
convincere Teonoe ( $\alpha \nu \alpha \nu \alpha \pi \varepsilon i \sigma \alpha \mu \varepsilon \nu$, ripreso poco più avanti con $\pi \varepsilon i \sigma \alpha \nu \tau \varepsilon$ al v. 828) con la supplica, ${ }^{63}$ e poi escogitare un piano di salvezza (v. 1034: $\mu \eta \chi \alpha \nu \eta \eta \nu \sigma \omega \tau \eta p i \alpha \varsigma$, con una ripresa del sostantivo usato al v. 813) ai danni di Teoclimeno.

La prima parte del piano -convincere Teonoe- raggiunge senza troppa difficoltà il suo scopo perché la sacerdotessa accoglie la supplica di Elena e la richiesta congiunta ${ }^{64}$ di Menelao con un argomento che contribuisce ad orientare il séguito del dramma in direzione del rispetto della giustizia e della corretta interpretazione del rapporto col sacro: nelle parole della sacerdotessa, non restituire Elena al marito sarebbe infatti un'ingiustizia ( $\dot{\alpha} \delta$ เ

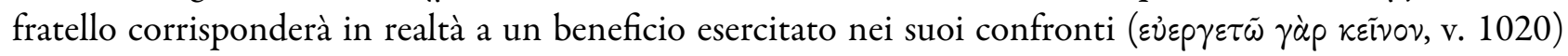
perché svilupperà in Teoclimeno un passaggio dalla mancanza di rispetto del sacro a una condizione di chi

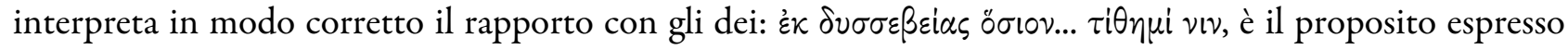
dalla sacerdotessa al v. 1021.

Sono almeno due gli elementi da osservare nella ideazione dell'inganno: da una parte, la scelta della persuasione come strategia che costruirà il piano di fuga, progressivamente irretendo e delegittimando Teoclimeno; dall'altra, l'affermazione di un principio di giustizia che si accompagna al ristabilimento di un corretto rapporto con il sacro, tanto più interessante perché si realizza attraverso la simulazione di un rito, fasullo nel complesso, ma ritualmente plausibile nei particolari. Questi elementi si intrecciano variamente 
nella tragedia, generando ulteriori linee di forza e di equilibrio che da questi dipendono, come il progressivo assorbimento di Menelao nella tessitura concreta dell'inganno.

\section{Due diversi inviti all'ascolto}

Un primo snodo nel dramma interviene dopo che Elena prende atto del successo incamerato ottenendo

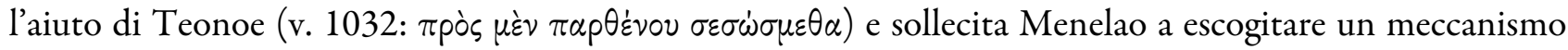
(v. 1034: $\mu \eta \chi \alpha \nu \dot{\eta})$ per fuggire. Si tratta di una svolta enunciata esplicitamente con una locuzione avverbiale

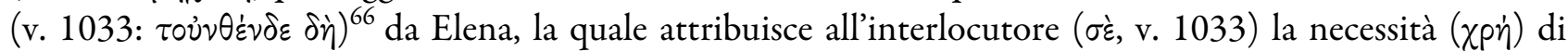

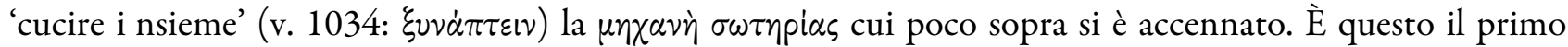
tentativo di coinvolgimento di Menelao, il quale reagisce alle parole di Elena in un modo che non produrrà alcun risultato, e servirà anzi solo per segnare un primo 'ingresso' dell'Atride nel meccanismo di inganno preso poi in mano, poco più avanti, da Elena.

Il passaggio è comunque interessante perché l'invito all'ascolto pronunciato da Menelao (v. 1035: äkove $\delta \dot{\eta} \nu v v)$ sembrerebbe annunciare una svolta nell'azione drammatica, collocandosi sulla stessa linea dell'invito all'ascolto pronunciato da Ifigenia quando ella inizia ad organizzare il piano di fuga dalla terra dei Tauri, chiedendo a Oreste e Pilade di ascoltarla ( $\dot{\alpha} \kappa o v \dot{\sigma} \alpha \tau \varepsilon$, IT 578). Si tratta tuttavia di un avvio fittizio, che poi non trova sbocco perché viene bloccato proprio da Elena che dissuade lo sposo dall'ipotesi di uccidere il sovrano, che egli torna ostinatamente a proporre (v. 1044) nonostante che già gli fosse stata identificata come non praticabile.

Secondo una linea analoga a quella che abbiamo già osservato per l'Ifigenia, viene cioè definendosi ancora

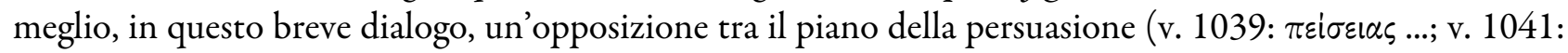
$\left.\pi \varepsilon i \sigma \alpha \mu \mu^{\prime} \ddot{\alpha} \nu\right){ }^{67}$ e l'ipotesi di una soluzione fondata sull'uso della forza (v. 1044: $\left.\kappa \tau \dot{\alpha} \nu \circ \mu^{\prime} \alpha^{\prime} \nu \alpha \kappa \tau \alpha\right)$ che deve fare i conti con l'impraticabilità di questa idea (già enunciata al v. 809) qui ribadita sulla base di una solidarietà sororale che, in fondo, lega Teonoe a Teoclimeno. A tutto questo si aggiunge anche un problema materiale

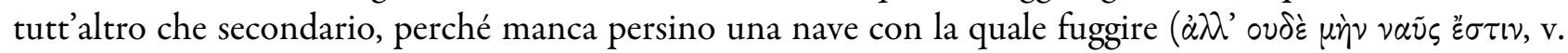
$1047) .^{68}$

L'idea alla quale Menelao ha chiesto di prestare ascolto si caratterizza dunque come aporetica in senso

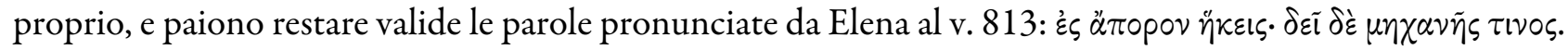

Di fronte ad una situazione che pare non avere sbocco, è allora Elena che invita l'interlocutore all'ascolto e, per così dire, avvia un primo ribaltamento dei rapporti di forza, prendendo in mano la situazione. È come se, passando dall'imperativo presente (äkove, v. 1035) con cui il marito suggerisce la propria inefficace strategia,

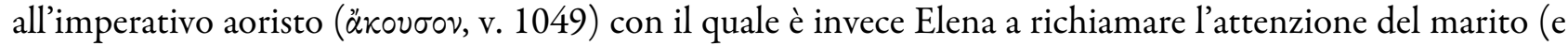
del pubblico), si registrasse anche un passaggio concreto da un'idea senza sbocco alla strategia che condurrà, al termine del dramma, alla partenza verso le coste di Nauplion e, da lì, verso Micene e Sparta. ${ }^{69}$

Gli elementi di contatto con l'Ifigenia sono molteplici: come si è detto, anche la figlia di Agamennone dà infatti avvio alla svolta nella costruzione dell' inganno con un richiamo all'ascolto enunciato con l'imperativo ákov่ $\alpha \tau \varepsilon$ (IT 578); anche in quel caso lo stratagemma viene identificato come 'femminile' (IT 1031-1032;

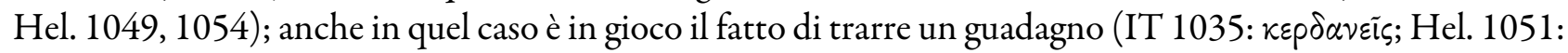
$\kappa \varepsilon p \delta \alpha \nu \tilde{\omega})$ da una situazione potenzialmente negativa che deve essere sfruttata. Più in particolare, nell' Elena si tratta di risultare «morto a parole» $\left(\lambda \dot{\partial} \gamma \omega \theta \alpha \nu \varepsilon \tilde{\varepsilon} \nu\right.$ v. 1054) ${ }^{70}$ e di trarre poi le conseguenze rituali di questa morte, con il rito tutto femminile del compianto (taglio dei capelli e lamentazione funebre) celebrato di

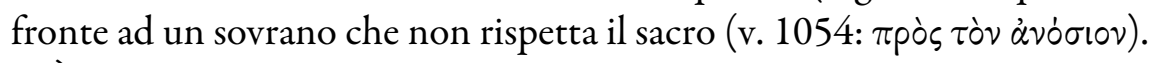

È come se, con il proprio intervento, Elena avviasse, tanto nel dialogo con Menelao quanto con la ṕñ dei vv. 1085-1106, la tessitura di fili che vengono per ora solo enunciati come progetto, e poi sono ripresi 
poco più avanti, al momento dell'entrata di Teoclimeno e dopo uno stasimo, il primo, che arriva dopo più di mille versi dall'inizio del dramma.

Con le proprie parole, la moglie di Menelao descrive al marito la gestualità funeraria consueta, fatta di gesti codificati e noti al pubblico sin dalle scene di lamentazione omeriche, che ella prima progetta e poi subito riprende quando, poco più avanti, annuncia il proprio rientro in casa (v. 1087: દ̇ solkovs $\beta \tilde{\alpha} \sigma \alpha)$ descrivendo ciascuna azione con maggiore grado di dettaglio: saranno tagliati i riccioli, sarà cambiato l'abito bianco in nero e sarà avviato il graffio delle guance fino a fare affiorare il sangue. ${ }^{71}$

Questi elementi di inganno saranno ripresi al momento dell'entrata di Teoclimeno, che mostra stupore di fronte ai pepli neri che hanno sostituito l'abito bianco (vv. 1186-87 $\pi \dot{\varepsilon} \pi \lambda \lambda_{0 \nu \varsigma} \mu \dot{\varepsilon} \lambda \alpha \nu \alpha \varsigma \lambda \varepsilon v \kappa \tilde{\omega} \nu$ che riprende $\pi \dot{\varepsilon} \pi \lambda \omega \nu \lambda \varepsilon v \kappa \tilde{\omega} \nu \mu \dot{\varepsilon} \lambda \alpha \nu \alpha \varsigma$ del v. 1088), ai capelli tagliati (v. 1187-88, con una ripresa del verbo $\dot{\varepsilon} \mu \beta \dot{\alpha} \lambda \lambda \omega$, che al v. 1089 era invece riferito al gesto di graffiarsi), e alle guance su cui scorrono lacrime (vv. 1189-90, con

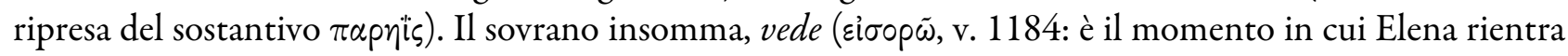
sulla scena) ciò che Elena ha prima progettato, e poi concretamente realizzato avviandosi in casa.

A differenza di questa parte del rito - che attinge a un patrimonio di competenze rituali condivise tra personaggi e pubblico- la seconda parte del piano non pare trovare riscontro nelle usanze funerarie elleniche, e prevede la realizzazione di un funerale in mare (v. 1062: $\pi \varepsilon \lambda \alpha \gamma i o v \varsigma \dot{\varepsilon} \varsigma \dot{\alpha} \gamma k \dot{\alpha} \lambda \alpha \varsigma$, un'espressione ripresa anche

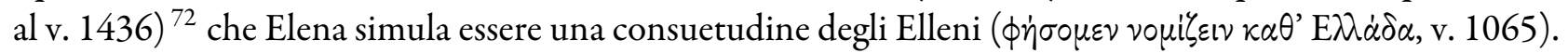

Il secondo elemento anticipato da Elena -il cenotafio annunciato al v. 1057 ( $\kappa \varepsilon \nu \tilde{\omega} \tau \dot{\alpha} \phi \omega)$ ) poi variamente ripreso da una marcata insistenza intorno al medesimo campo semantico nel giro di pochi versi (v.

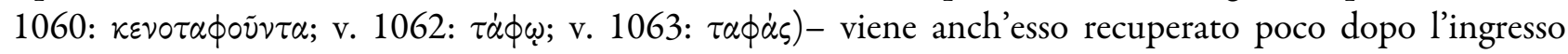
di Teoclimeno, quando il dialogo vira in direzione della costruzione concreta dell'inganno. Dopo avere raccontato la falsa notizia della morte di Menelao, Elena fa infatti riferimento alla mancata sepoltura del proprio sposo ( $\alpha \theta \alpha \pi \tau o v$, ripetuto ai vv. 1222 e 1223), con questo offrendo a Teoclimeno la possibilità di intuire, e addirittura anticipare, ${ }^{73}$ la spiegazione dell'avvenuto taglio dei capelli (in questo caso si riprende il termine $\beta \circ \sigma \tau$ ขن́xovs, usato qal v. 1224 e già al v. 1087, sempre in connessione con $\tau \dot{\varepsilon} \mu \nu \omega)$. La notizia della mancata sepoltura permette di riprendere il tema del rito funerario da celebrare che, nella sticomitia tra la protagonista e il sovrano, si configura come un inganno che parte dalla simulata approvazione, da parte della prima, delle nozze tanto agognate dal secondo (vv. 1231-32), il quale accetta così una riconciliazione e acconsente alla richiesta di seppellire il morto.

È un meccanismo, ad un tempo, semplice e complicato, quello che si avvia a questo punto del dramma, e vale la pena di riprenderne il filo.

\section{Un coinvolgimento progressivo}

Mentre prende finalmente atto del lutto, Teoclimeno inizia a nutrire il sospetto (vv. 1226-1228) che il pianto di Elena sia solo un modo per allontanare le nozze, mantenendosi fedele ( $\pi$ เ $\tau \dot{\eta}$, v. 1230) al marito e fuggendo $(\phi \varepsilon \dot{y} \gamma \sigma v \sigma \alpha)$ il sovrano. Tra i fili tessuti dalla sposa di Menelao, intrecciati in precedenza e qui recuperati, va registrata la ripresa del tema della sepoltura (tre volte in pochi versi: v. 1228, $\tau \dot{\alpha} \phi o v ;$ v. 1239, $\theta \dot{\alpha} \psi \alpha \iota ;$ v. $1240, \theta \dot{\alpha} \psi \varepsilon \iota s)$, qui associato a quello della costruzione di una tomba per il defunto (vv. 1240, 1244), cruciale per il rito funerario.

Qui sta l'inganno, e qui sta un ulteriore elemento di analogia con quanto accade nella Ifigenia tra $i$ Tauri, perché Teoclimeno garantisce alla sua interlocutrice la possibilità di fruire degli spazi che ella desidera,

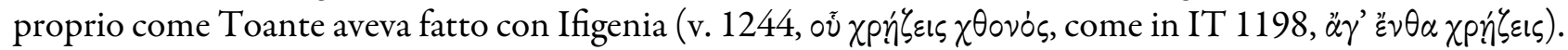
Se tuttavia nella terra dei Tauri la sorella di Oreste cerca un luogo isolato nei pressi del mare, in questo caso Elena gioca sulla determinazione di luogo ( $\chi$ Өovós) per precisare che non è quello il modo in cui i Greci seppelliscono ( $\tau \mu \beta \varepsilon v \dot{\nu} о \mu \varepsilon v$, è il verbo usato al v. 1245) i morti in mare. Viene tra l'altro recuperato anche il 


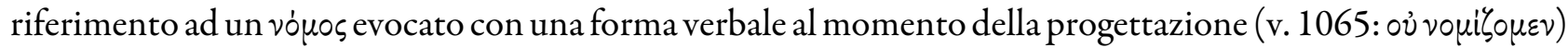
e qui ripreso in forma nominale (v. 1246: $\tau \tilde{\omega} \nu \nu \delta \mu \omega \nu)$, con lo scopo di mostrare l'estraneità di Teoclimeno $\mathrm{e}$, a dire il vero, anche del pubblico- a quel tipo di consuetudine.

Il sovrano egizio chiede perciò come, allora, $(\pi \tilde{\omega} \varsigma \delta \alpha i, \mathrm{v} .1246)^{74}$ si svolga il rito, dato che egli 'non riesce a stare al passo' ( $\lambda \dot{\varepsilon} \lambda \varepsilon \iota \mu \mu \alpha)$ delle usanze elleniche.

Una simile ammissione di ignoranza e richiesta di istruzioni costituisce un ulteriore snodo nella costruzione di un percorso che affianca la progressiva delegittimazione del sovrano a vantaggio di una crescita di autonomia dei due personaggi in fuga. La domanda di Teoclimeno apre cioè, di fatto, uno spiraglio nelle pieghe del quale Elena si insinua per coinvolgere direttamente Menelao nella costruzione dell'inganno, prima dichiarando la propria inesperienza ( $\dot{\varepsilon} \gamma \dot{\omega} \delta^{\prime} \ddot{\alpha} \pi \varepsilon ı \rho \varsigma$, v. 1249) di fronte alla ulteriore richiesta di dettagli su $\operatorname{cosa}(\tau i$ $\sigma \mathrm{l}, \mathrm{v} .1248)$ il sovrano debba preparare per il morto, e poi lasciando scivolare l'interlocuzione verso il proprio sposo, al quale viene ora rivolta da parte di Teoclimeno la medesima domanda che pochi versi prima

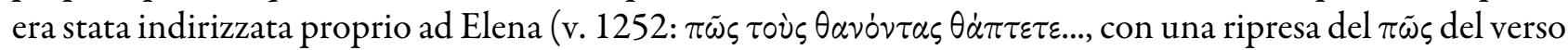
1246).

Dopo avere proposto una soluzione aporetica quando la moglie gli aveva chiesto di trovare uno stratagemma, ora Menelao si trova progressivamente coinvolto nella costruzione dell'inganno secondo la linea proposta da Elena, fondata sulla persuasione. Essendosi guadagnato la fiducia di Teoclimeno, Menelao si trova così nella condizione di poter guidare in prima persona la costruzione della $\mu \eta \chi \alpha \nu \eta \dot{\eta}$, in una sequenza di istruzioni rituali dettagliate, fino al limite quasi valicato del didascalico.

Lo strano $-\mathrm{e}$ non attestato presso i Greci- ${ }^{75}$ rito funerario marino è, per così dire, fabbricato con elementi coerenti con le pratiche funerarie elleniche, ed è articolato in momenti precisi, contenuti nella parte di sticomitia che coinvolge il pretendente sposo e il reale marito di Elena ai vv. 1251-77. Al primo che chiede dettagli, il secondo parla di un funerale il cui sfarzo deve corrispondere al patrimonio del morto

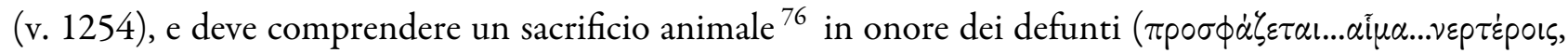
v. 1255), l'uso di un giaciglio per il cadavere ( $\sigma \tau \rho \omega \tau \dot{\alpha} \lambda \dot{\varepsilon} \kappa \tau p \alpha$, v. 1261), la consacrazione di armi di bronzo conformi allo statuto del guerriero (v. 1263), e un'offerta di frutti della terra (v. 1265). L'insieme dell'intero sacrificio -aggiunge infine l'Atride- dovrà svolgersi lontano dalla riva ( $\chi \varepsilon \rho \sigma \dot{\theta} \theta \varepsilon v$, v. 1269), per una usanza

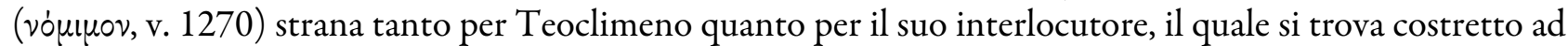
inventare un'implausibile ragione, connessa con l'opportunità che gli scarti del sacrificio (v. 1271: $\lambda \dot{u} \mu \alpha \tau \alpha)$ non tocchino terra. ${ }^{77}$

Il rito è poi ricondotto nel binario della credibilità, anche agli occhi del pubblico, quando Menelao ricorda la peculiarità familiare del rito funerario, che deve essere celebrato 'dalla madre, dalla moglie o dai figli' (v. 1275).

L'intera scena si costruisce intorno all'intreccio tra la linea della necessità (fittizia, ma credibile) di rispettare una consuetudine più volte indicata come tale da parte dei fuggitivi, e la linea dell'accettazione di questa consuetudine da parte di un Teoclimeno che proclama a più riprese la propria ignoranza e si affida ai suoi interlocutori quando, suo malgrado, accetta lo svolgimento del funerale marino, pur opponendo resistenza su singoli punti.

Uno di questi, in particolare, merita attenzione.

La specificazione relativa alla peculiarità familiare di coloro che possono partecipare al rito funerario dipende dalla richiesta, avanzata proprio dal sovrano egizio, che Menelao svolga autonomamente il rito funerario (vale a dire senza Elena: $\chi \omega$ pis $\tau \tilde{\eta} \sigma \delta \varepsilon$, v. 1274), richiesta alla quale l'Atride ha buon gioco a rispondere nel modo - ritualmente più che credibile -che si è appena detto, richiamando cioè l'opportunità che siano madre, moglie e figli a occuparsi del rito. L'Atride dà così la possibilità a Teoclimeno di sottolineare come

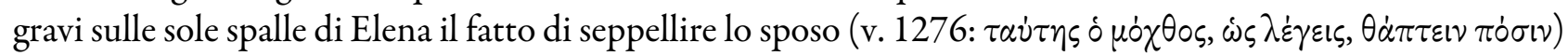
e ha così l'occasione di ribadire la necessità di non privare dei riti funerari $(\nu \dot{\jmath} \mu \mu \alpha)$ i morti, qualora si voglia

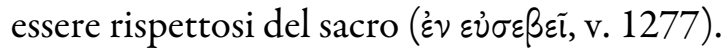


Anche quest'ultimo punto è importante.

Si è già avuto modo di osservare, infatti, quanto il tema del rispetto del sacro sia cruciale per la costruzione, e soprattutto per lo sviluppo, della figura drammatica di Teoclimeno. Si ricorderà che, al momento di accordare ad Elena e a Menelao il proprio silenzio e la propria collaborazione (vv. 998-1029), Teonoe aveva specificato il desiderio di fare passare il fratello dalla condizione di chi non rispetta il sacro ( $\dot{\kappa} \kappa \delta v \sigma \sigma \varepsilon \beta \varepsilon i \alpha \varsigma)$ a quella, opposta, di chi interpreta in modo corretto il rapporto con gli dei (ö $\sigma \circ \nu . . . \tau i \theta \eta \mu t$ $\nu$ vv, v. 1021). Appare allora ancora più significativo il passaggio di cui ci stiamo ora occupando, tanto per le parole di Menelao al v. 1277, quanto per la reazione immediata di Teoclimeno, il quale individua proprio nel rispetto del sacro la caratteristica per lui più importante in colei che egli si ostina a qualificare come

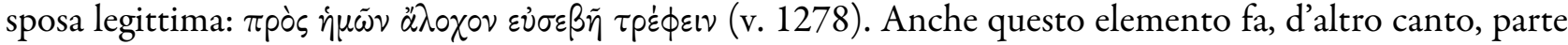
dell'inganno. È stata infatti Elena ad avere proposto al sovrano di dimenticare il passato e, soprattutto, ad averlo autorizzato a dare avvio ( $\alpha \chi \chi \omega$ è il verbo utilizzato al v. 1231) alle cerimonie nuziali ( $\tau \tilde{\omega} \nu \dot{\varepsilon} \mu \tilde{\omega} \nu$ $\gamma \dot{\alpha} \mu \omega \nu)$, un dato che rimane per ora sospeso, ma tornerà più avanti come elemento di rilievo. Limitiamoci, per ora, ad averlo identificato.

\section{L'inganno, finalmente (ancora una volta)}

Anche nell'Elena la preparazione concreta dell'inganno avviene in uno spazio chiuso ${ }^{78}$ nel quale si realizza ciò che è stato preparato a voce: Teonoe mantiene il segreto, ingannando il fratello che le chiede dettagli, e Menelao prepara le armi necessarie per il simulato omaggio al morto.

È Elena a raccontare ciò che è accaduto nel palazzo, una volta rientrata sulla scena al v. 1369 e prima dell'ingresso di Teoclimeno al v. 1385. Questa volta l'insistenza di Elena è sulla svolta positiva del destino

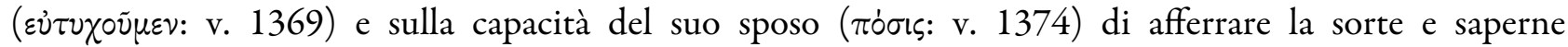
approfittare (v. 1374). Il dettaglio è importante, tanto dal punto di vista drammaturgico (le armi saranno essenziali quando l'inganno sarà svelato), quanto -ed è il particolare che più ci interessa- dal punto di vista dello sviluppo del personaggio di Menelao, che continua ad assumere un peso via via crescente nella costruzione e nella gestione del piano di fuga.

Viene cioè sviluppandosi un doppio movimento che marca, da una parte, la progressiva perdita di autonomia e autorità da parte di Teoclimeno e, dall'altra, una corrispondente acquisizione di incisività sugli eventi da parte di Menelao. Anche in questo processo, il riferimento alle azioni rituali gioca un ruolo cruciale, tanto nella costruzione del contenitore all'interno del quale viene gestita l'intera trappola (il simulato rito funerario), quanto nella determinazione dei rapporti di forza e nella possibilità, per i diversi personaggi, di diventare motore degli eventi o di subirne lo svolgimento, fino ad una presa di coscienza di cui tutti paiono rendersi conto tranne, come è ovvio, il personaggio ingannato.

Teoclimeno oscilla tra il rispetto e la trasgressione di una norma rituale che egli fatica ancora ad accettare completamente. Dopo la descrizione, da parte di Elena, dei preparativi e la richiesta di complicità del coro ${ }^{79}$ il sovrano egizio dà infatti addirittura l'ordine ai propri servi di seguire le istruzioni rituali (vv.

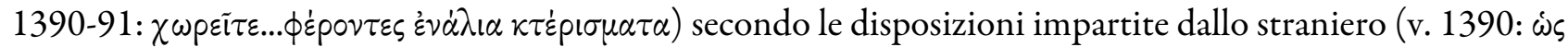
$\ddot{\varepsilon} \tau \alpha \xi \varepsilon \nu \delta \xi \xi \dot{\varepsilon} \nu \circ \varsigma)$, con questo di fatto accrescendo ulteriormente la legittimazione di Menelao come parte attiva nella $t$ essitura dell'inganno. Al tempo stesso, Teoclimeno continua però a resistere di fronte ad un'accettazione passiva della consuetudine rituale, tornando a chiedere alla protagonista del dramma

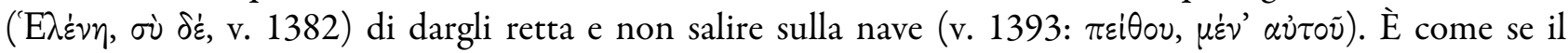
sovrano tentasse ora di invertire i 1 corso di eventi che sin dall'inizio sono stati invece impostati dalla sua interlocutrice per svolgersi secondo una direzione contraria sulla quale abbiamo già insistito più volte: in questo caso il sovrano chiede che il processo indicato da $\pi \varepsilon i \theta \varepsilon \iota \nu$ inverta per così dire il suo corso, e sia addirittura Elena a lasciarsi persuadere da 1 ui, e non l'inverso. 
La risposta di Elena ai vv. 1399-410 è di nuovo agganciata alla necessità di rispettare l'onore dovuto al morto (v. 1404: $\dot{\varepsilon} v \tau \dot{\alpha} \phi i \alpha),{ }^{80}$ ed è associata a un richiamo verbale utile a porre l'accento sull'inganno subito da

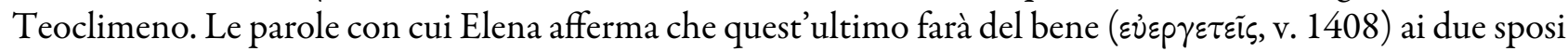
comportano infatti una ripresa del verbo $\varepsilon \dot{\varepsilon} \varepsilon \gamma \varepsilon \tau \dot{\varepsilon} \omega$ usato da Teonoe quando ella aveva affermato che, pure

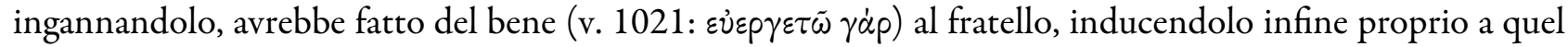
rispetto del sacro sul quale abbiamo già avuto modo di insistere. ${ }^{81}$

Il ruolo di Menelao diviene via via più importante e, a differenza di quel che accade nella Ifigenia, è piuttosto sul versante maschile della coppia (lì di fratelli, qui di coniugi) che, a partire da questo momento, ricade il peso della realizzazione dell'inganno, non tanto per quel che riguarda la descrizione della lotta con l'esercito locale (in entrambe le tragedie ovviamente condotta dagli uomini), quanto nella legittimazione all'esercizio dell'autorità nella concretizzazione del piano così a lungo tessuto da Elena. L'irretimento di Teoclimeno passa infatti anche per una serie di richieste della protagonista del dramma, la quale prima si informa se a

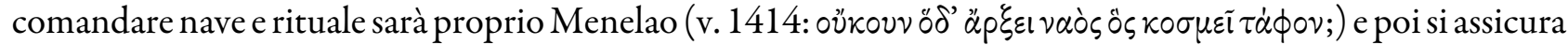

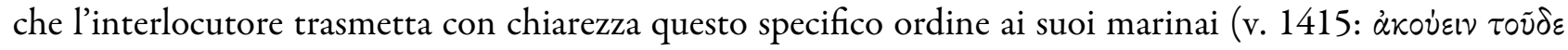

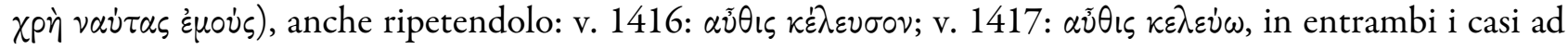
inizio di verso.

Il sovrano è dunque ingannato a tal punto da riprendere verbatim le parole di Elena, che esprime un

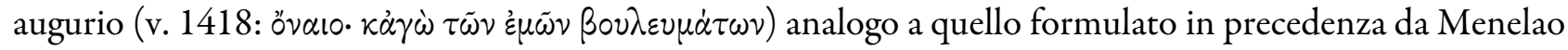

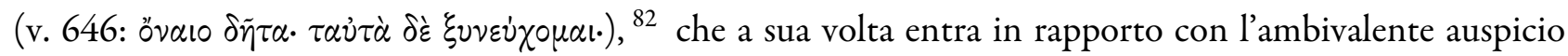

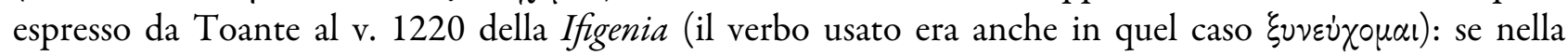
tragedia ambientata tra i Tauri l'unirsi all'augurio del sovrano era uguale e contrario a quello espresso dalla protagonista affinché il rituale andasse bene, anche in questo caso Elena e Teoclimeno desiderano cose opposte, ma qui la protagonista recupera - con la ripresa di ővaıo- la formulazione del medesimo desiderio espresso dal marito. A questo si aggiunge un progressivo trasferimento di autorità da Teoclimeno a Menelao, al quale ora i marinai devono ubbidire, come Elena chiede sia detto e ribadito con chiarezza proprio dal sovrano (iv $\sigma \alpha \phi \tilde{\omega} s \mu \dot{\alpha} \theta \omega \sigma i$ бov, v. 1416).

A completare il quadro dell'inganno sta lo scambio di battute ulteriore, nel quale Teoclimeno prova un'ultima volta a proporsi come guida della spedizione in mare (v. 1427), ${ }^{83}$ ma Elena risponde con una formulazione che vorrebbe valorizzare l'autorità di Teoclimeno, e finisce invece per enfatizzare il ruolo

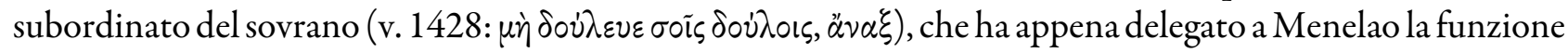
di comando.

Va inoltre ricordata anche la reazione del personaggio ingannato, il quale finisce per dirottare nella direzione sbagliata l'autorità rituale della quale egli cerca di investirsi.

Viene infatti qui recuperato il filo tematico dei riti nuziali incoraggiati da Elena al v. 1231, e poi di fatto rimasto in sospeso. Al v. 1431 Teoclimeno ordina che qualcuno vada a dire ("i $\tau \omega \delta \dot{\varepsilon} \tau\llcorner\varsigma \phi \rho \dot{\alpha} \sigma \omega \nu$ ) alle sole persone sulle quali egli possa ancora esercitare autorità (v. 1432: vं

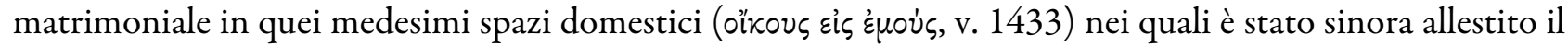
simulato rito funerario.

Si tratta cioè di portare all'interno della casa tutto ciò che può rendere degne d'ammirazione le nozze $(\dot{\alpha} \gamma \dot{\alpha} \lambda \mu \alpha \tau \alpha \ldots \gamma \dot{\alpha} \mu \omega \nu, \text { v. } 1433)^{84}$ e di allestire un rito che sia riconoscibile all'esterno della casa per via uditiva: bisogna infatti ( $\chi \rho \dot{\eta}$, v. 1433) che tutto il territorio risuoni del canto degli inni e dell'imeneo (vv. 1434-35), con il preciso scopo di destare invidia in chi ascolta ( $\dot{\omega} \zeta \eta \lambda \omega \tau$ òs $\eta \tilde{\eta}$, v. 1435).

Funge insomma da cassa di risonanza per l'inganno questa prospettiva di festa immaginata, pensata e progettata da Teoclimeno, il quale oscilla tra l'accettazione di un rito che non conosce e non capisce, ma di cui intuisce il potenziale rischio per il proprio desiderio di sposare Elena, e la frustrazione per un'autorità e una conseguente guida dell'azione drammatica che non può invece esercitarsi su ciò che sta effettivamente 
accadendo sulla scena. Al sovrano egizio non resta, quindi, che immaginare una festa nuziale come forma rituale legittimante l'unione auspicata, ma destinata solo ad amplificare le sue frustrazioni fino al limite della ridicolizzazione del personaggio, quando egli addirittura invita Menelao a prendere parte al banchetto di nozze ( $\sigma \nu v \delta \alpha i \sigma \alpha \varsigma$, v. 1439), una volta che l'Atride sarà rientrato dallo svolgimento del simulato rito funerario in mezzo al mare ( $\pi \varepsilon \lambda \alpha \gamma i$ ov $\left.\dot{\varepsilon}_{\varsigma} \dot{\alpha} \gamma \kappa \dot{\alpha} \lambda \alpha \varsigma\right)$.

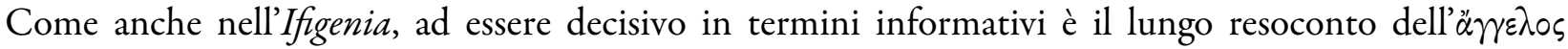
(vv.1526-1618), nel quale vengono raccontati tutti i passaggi che vanno dall'allestimento della nave (descritto con un notevole grado di precisione), ${ }^{85}$ all'imbarco di una notevole quantità di guerrieri, fino alle difficoltà di fare salire un toro riluttante (vv. 1555-59) e all'agilità con cui l'animale viene poi caricato dai marinai

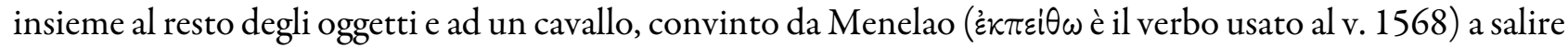
sull'imbarcazione.

\section{$\Delta \dot{\partial} \lambda \operatorname{los} \dot{\eta} \nu \alpha \nu \kappa \lambda \eta p i \alpha$}

La lunga descrizione della lotta a bordo della nave si accompagna ad un dettaglio che accomuna la realizzazione di questo inganno e quella orchestrata ai danni di Toante, perché anche in questo caso gli schiavi hanno maggiore consapevolezza di quel che sta accadendo, rispetto a quanta ne abbia il sovrano locale ingannato. Anche qui, infatti, al sovrano viene rivelato che gli schiavi avevano in effetti maturato un

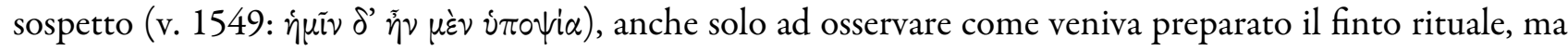

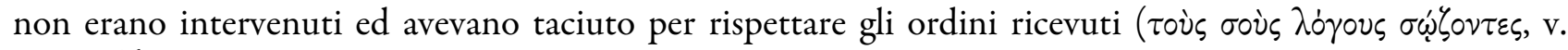
1552). ${ }^{86}$ La progressiva delega di autorità che Teoclimeno ha accettato finisce così per rivoltarsi contro di lui: si è visto con quanta cura Elena abbia chiesto e ottenuto che i marinai accettassero di seguire le istruzioni di uno straniero al quale lo stesso sovrano si era rivolto per sapere come dovesse svolgersi lo strano rito marino. Anche in questo caso la responsabilità viene addossata al sovrano, con un recupero del tema del trasferimento di autorità verso Menelao. ${ }^{87}$

La conclusione è poco più di una constatazione, da parte di marinai che finiscono per assumere essi stessi il comando (v. 1590: $\dot{\alpha} \xi i \omega \varsigma$ $\kappa \dot{\varepsilon} \lambda \varepsilon v \varepsilon \sigma \dot{v}$, con un recupero del verbo $\kappa \varepsilon \lambda \varepsilon \dot{\omega} \omega$, questa volta riferito ai marinai) quando realizzano che tutto ciò cui essi stavano assistendo era solo un'evidente simulazione.

La formulazione usata dai marinai è analoga a quella usata nell'Ifgenia a proposito dei $\kappa \alpha \theta \dot{\alpha} p \mu \alpha \tau \alpha$ definiti $\delta \dot{\partial} \lambda \iota \alpha$. Se lì era la purificazione ad essere un tranello, qui è la spedizione navale a risultare un inganno a chi abbia avuto occhi per vedere: $\delta \dot{\lambda} \lambda \iota_{1} \varsigma \dot{\eta} v \alpha v k \lambda \eta p i \alpha$, sono infatti le parole usate da uno dei marinai al v. 1549 , quando ormai non resta che prendere atto di ciò che Teoclimeno non ha saputo comprendere. Come Toante, anche il sovrano egizio si rende conto troppo tardi della trappola in cui è stato preso per mano di Teonoe e di

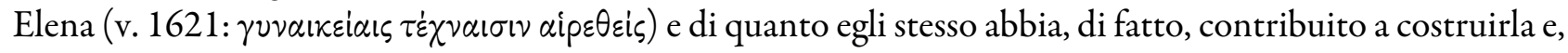
poi, anche a realizzarla attraverso una progressiva autoemarginazione e un crescente trasferimento di autorità a favore di Elena, di Menelao e anche dei propri marinai.

Prima dell'intervento dei Dioscuri ex machina (vv. 1642-79) c'è un ultimo passaggio che merita di essere osservato, e riguarda il comportamento del gruppo corale, che tenta in ogni modo di bloccare Teoclimeno (oن̉火 $\dot{\alpha} \phi \dot{\eta} \sigma o \mu \alpha \iota ~ \pi \dot{\varepsilon} \pi \lambda \omega \nu \sigma \tilde{\omega} \nu$, v. 1629) ${ }^{88}$ che vuole uccidere la sorella, ritenendo di rispondere con questo

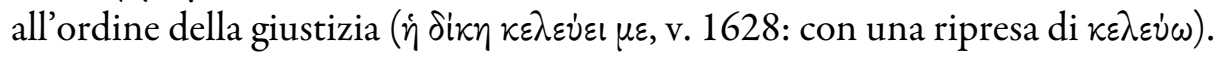

Ad interessarci, per concludere, è ciò che i due interlocutori si dicono nel verso 1638, perché il sovrano ha una sorta di ultimo sussulto relativo al manifestarsi della propria autorità, che finirebbe per essere addirittura

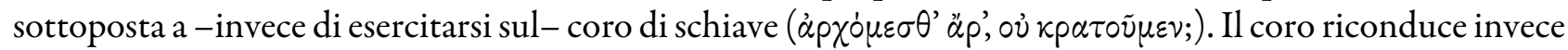

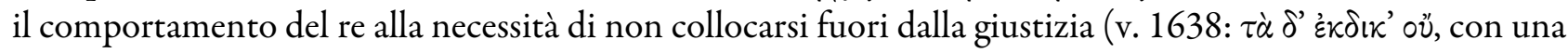
ripresa di quanto già detto al v. 1628), ma anche di mantenere nel giusto equilibrio il rapporto con gli dei e con le consuetudini sacre ("̋ $\sigma\llcorner\delta \rho \tilde{\alpha} \nu)$. 
Come abbiamo avuto modo di ribadire più volte, è esattamente ciò che ha inteso fare la sorella quando ha accettato di aiutare Elena e Menelao, ed è ciò che è stato richiamato a più riprese per costruire la necessità di svolgere un rituale funerario fasullo, ma evidentemente efficace.

\section{RIFERIMENTI}

Barber, E.J.W. (1992). Images of Athena on the Akropolis. En J. Niels (Ed.), Goddess and Polis. The Panathenaic Festival in Ancient Athens (pp. 119-42). Hanover: Princeton University Press.

Basta Donzelli, G. (1978). Studio sull'Elettra di Euripide. Catania: Pubblicazioni della Facoltà di Lettere e Filosofia.

Basta Donzelli, G. (1995). Euripides. Electra. Stutgardiae et Lipsiae: Teubner.

Beaulieu, M.-C. (2019). $\Theta \varepsilon \tilde{\omega} \nu \check{\alpha} \gamma \nu i \sigma \mu \alpha \mu \dot{\varepsilon} \gamma\llcorner\sigma \tau o v$ : la mer et la purification en Grèce Ancienne. En J. M. Carbon e S. Peels-Matthey (Eds.) Kernos Supplement 32. Purity and Purification in the Ancient Greek World. Texts, Rituals, and Norms (pp. 207-24).

Bendlin, A. (2008). Purity and Pollution. En D. Ogden (Ed.) A Companion to Greek Religion (pp. 178-189). Oxford: Blackwell.

Boegehold, A. (1995). The Lawcourts of Athens. Sites, Buildings, Equipments, Procedures, and Testimonia. Princeton: The Athenian Agora, vol. XXVIII.

Bremmer, J. (2007). Greek Normative Animal Sacrifice. En D. Ogden (Ed.), A Companion to Greek Religion (pp. 132-144). Oxford: Blackwell.

Burkert, W. (2003). La religione greca. Milano: Jaca Book.

Cerri, G. (1996). Omero. Iliade. Milano: Rizzoli.

Cohen, D. (1983). Theft in Athenian Law. München: Beck.

Connelly, J. B. (2007). Portrait of a Priestess. Women and Ritual in Ancient Greece. Princeton: Princeton University Press.

Corbett, P. E. (1970). Greek Temples and Greek Worshippers: The Literary and Archaeological evidence, BICS, 17, $149-58$.

Cropp, M. J. (1988). Euripides. Electra. Warminster: Aris \& Phillips.

Denniston, J. D. (1939). Euripides Electra. Oxford: Clarendon Press.

Denniston, J. D. (1954). The Greek Particles. Oxford: Oxford University Press.

Di Benedetto, V. y Medda, E. (1997). La tragedia sulla scena. La tragedia greca in quanto spettacolo teatrale. Torino: Einaudi.

Distilo, N. (2012). Commento critico-testuale all'Elettra di Euripide. Padova: S.A.R.G.O.N.

Donohue, A. A. (1988). Xoana and the origin of Greek Cults. Oxford: Oxford University Press

Ellinger, P. (2021). Enlèvements de statues divines en bord de mer: de l'Artémis taurique à Héra Reine, História: Questões \& Debates, 69(1), 163-82.

Elmsley, P. (1813). Euripidis Heraclidae. Oxonii: Collingwood.

Fletcher, J. (2003). Women and Oaths in Euripides, Ĺeatre Journal, 55(1), 29-44. https://doi.org/10.1353/tj.200 3.0020

Fletcher, J. (2012). Performing Oaths in Classical Greek Drama. Cambrige: Cambridge University Press

Fletcher, J. (2017). Euripides and Religion. En L. K. McClure (Ed.) A Companion to Euripides (pp. 483-99). Chichester: Blackwell.

Frisone, F. (2000). Leggi e regolamenti funerari nel mondo greco. I. Le fonti epigrafiche. Galagina: Congedo Editore.

Giordano, M. (1999). La supplica. Rituale, istituzione sociale e tema epico in Omero, Roma: AION Sezione filologicoletteraria.

Hall, E. (1989). Inventing the Barbarian: Greek Self-Definition through Tragedy. Oxford: Oxford University Press. 
Kubo, M. (1967). The Norm of Myth: Euripides' Electra, HSCPh, 71, 15-31.

Larson, J. (2007). Ancient Greek Cults: a guide. New York - London: Routledge.

Lupu, E. (2005). Greek Sacred Law. Leiden: Brill.

Luschnig, C. A. E. y Roisman, H. M. (2011). Euripides. Electra. A Commentary. Norman OK: University of Oklahoma Press.

Mastronarde, D. J. (1979). Contact and Discontinuity. Some Conventions of Speech amd Action on the Greek Tragic Stage. Berkeley-Los Angeles CA: University of California Press.

Mac Dowell, D. (1963). Athenian Homicide Law in the Age of the Orators. Manchester: Manchester University Press.

Medda, E. (2007). La casa di Elettra. Strategie degli spazi e costruzione del personaggio nelle due Elettre, Dioniso n.s. VI, 44-67 (= La saggezza dellillusione. Studi sul teatro greco. Pisa: ETS 2013, 81-112).

Medda. E. (2017). Eschilo. Agamennone. 3 voll. Roma: Bardi.

Meinel, F. (2015). Pollution and Crisis in Greek Tragedy. Cambridge: Cambridge University Press.

Mirto, M. S. (1980). Il sacrificio tra metafora e mechanema nell'Elettra di Euripide, CCC, 1, 299-331.

Mugelli, G. (2019). «La flamme dévoratrice d'offrandes»: feu et agentivité rituelle dans la tragédie grecque, Cabiers Mondes anciens, 12, http://journals.openedition.org/mondesanciens/2406

Naiden, F. (2006). Ancient Supplication. Oxford: Oxford University Press.

Osborne, R. (1993). Women and Sacrifice in Classical Greece, CQ, 43, 392-405.

Palmisciano, R. (2017). Dialoghi per voce sola. La cultura del lamento funebre nella Grecia antica, Roma: Quasar.

Parker, R. P. (1983). Miasma. Pollution and Purification in early Greek Religion. Oxford: Clarendon.

Parker, R. P. (2005). Polytheism and Society at Athens. Oxford: Oxford University Press.

Parker, R. P. (2011). On Greek Religion. Ithaka: Cornell University Press.

Parker, R. P. (2018). Miasma: Old and New Problems. En J.-M. Carbon y S. Peels-Matthey (Eds.) Kernos Supplement, 32, Purity and Purification in the Ancient Greek World. Texts, Rituals, and Norms (pp. 23-34).

Parker, L. P. E. (2016). Euripides' Iphigenia in Tauris. Oxford: Oxford University Press.

Roisman, H. M. (2017). Electra. En L. K. McClure (Ed.) A Companion to Euripides (pp. 166-181). Chichester: Blackwell.

Schwinge, E. R. (1968). Die Verwendung der Stichomythie in den Dramen des Euripides. Heidelberg: Winter.

Seidler, A. (1813). Euripidis Electra. Lipsiae: Fleischer.

Sourvinou-Inwood, Ch. (1996). 'Reading' Greek Death: To the End of the Classical Period. Oxford: Oxford University Press.

Stengel, P. (1894). v. Amphidromia in RE 1, coll. 1901-1902.

Stevens, P. T. (1976). Colloquial expressions in Euripides (Hermes, Einzelschriften, 38). Wiesbaden: Steiner.

Taddei, A. (2009). Inno e pratiche rituali in Euripide. Il caso della Ifigenia tra i Tauri, PAIDEIA, 64, 235-53.

Taddei, A. (2020). Heortè. Azioni sacre sulla scena tragica euripidea. Pisa: ETS.

Taddei, A. (2021). Tra Omero, i tribunali ed il teatro. Giuramenti tra oralità e scrittura in Grecia antica. En S. Baggio y A. Di Ricco. Immagini della scrittura e metafore dell'atto creativo. Trento: Università di Trento, Labirinti.

Todd, S. C. (1993). The Shape of Athenian Law. Oxford: Oxford University Press.

Todd, S. C. (2007). A Commentary on Lysias'Speeches 1-11. Oxford: Oxford University Press.

Torrance, I. (2009). On your head be it sworn: oath and virtue in Euripides Helen, CQ, 59, 1-7.

Van Straten, F. T. (1995). Hierà Kalà : Images of Animal Sacrifice in Archaic and Classical Greece. Leiden: Brill. 


\section{Nota}

* Questo lavoro è stato sviluppato e discusso in ogni sua parte da entrambi gli autori; nello specifico, il paragrafo sull'Elettra è di Enrico Medda, e i paragrafi sulla Ifigenia Taurica e l'Elena di Andrea Taddei.

1 Un breve panorama sui principali orientamenti della critica si può leggere in Taddei, 2020, pp. 17-21, con bibliografia ulteriore.

2 Il testo dell'Elettra è citato secondo l'edizione di Basta Donzelli (1995).

3 Sulla morte sacrificale di Egisto si veda soprattutto l'ampia discussione di Mirto (1980, pp. 306-12).

4 Alcune leggi sacre relative a diverse regioni, menzionate da Parker (1983, p. 50 n. 67), divergono circa il numero di giorni dopo i quali chi era entrato in contatto con una partoriente recuperava la propria purezza: 2, 3, 7 o anche 9.

5 Per un quadro completo delle fonti sugli ả $\mu \phi i \delta \rho \dot{\mu} \mu \alpha$ cf. Stengel (1894, coll. 1901-1902).

6 Una rassegna delle diverse interpretazioni è offerta da Parker (1983, p. 51 n. 71). L'attribuzione alla cerimonia degli $\dot{\alpha} \mu \phi i \delta \rho \dot{\mu} \mu \alpha \alpha$ anche dell'assegnazione del nome al bambino, che si legge in alcuni testimoni (ad esempio schol. Aristoph. Lys. 757 Hangard), è evidentemente frutto di confusione rispetto al rito della $\delta \varepsilon \kappa \dot{\alpha} \tau \eta$, cf. la nota seguente.

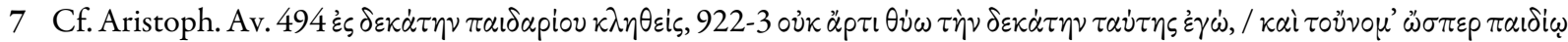

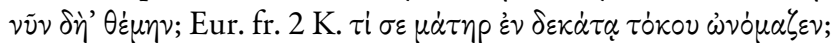

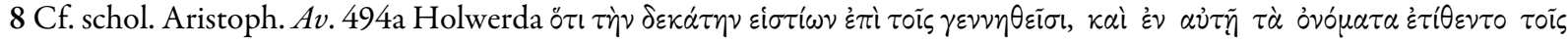

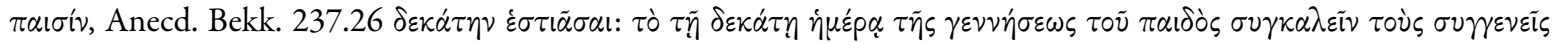

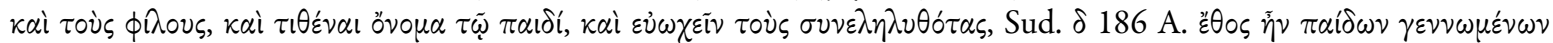

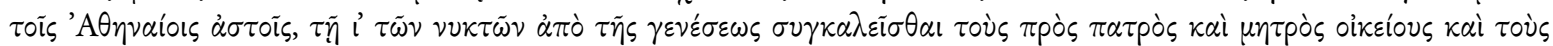

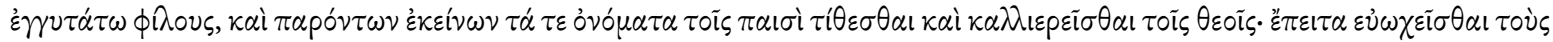

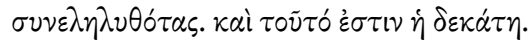

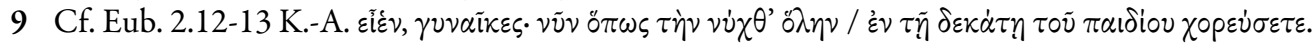

10 L'accusativo $\delta \varepsilon \kappa \dot{\alpha} \tau \eta \nu$ $\sigma \varepsilon \lambda \eta \dot{\nu} \eta \nu$ è ottima correzione di Musgrave, che restituisce alla frase un costrutto analogo a quello di Aristoph. $A v .922 \theta \dot{v} \omega \tau \dot{\eta} \nu \delta \varepsilon \kappa \dot{\alpha} \tau \eta \nu$ e ripristina il riferimento diretto alla pratica rituale della $\delta \varepsilon \kappa \dot{\alpha} \tau \eta$. Per altre, meno soddisfacenti interpretazioni della paradosi cf. Distilo, $2012 \mathrm{ad}$ l. L'uso di $\sigma \varepsilon \lambda \eta \dot{\nu} \eta$ per indicare le notti invece dei mesi è

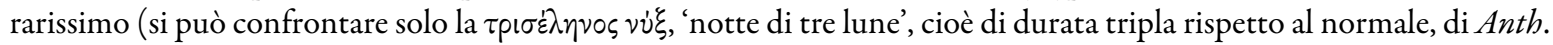
Gr. 16.102.1). Non c'è dubbio comunque che questo sia il significato necessario nel passo euripideo.

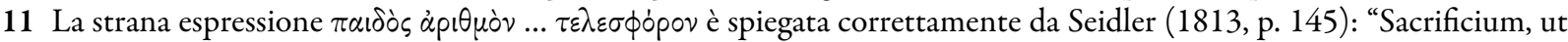
supra dictum est, vocabatur $\delta \varepsilon \kappa \dot{\alpha} \tau \eta ~ \sigma \varepsilon \lambda \dot{\varepsilon} \nu \eta$, vel simpliciter $\delta \varepsilon \kappa \dot{\alpha} \tau \eta$. Haec $\delta \varepsilon \kappa \dot{\alpha} \tau \eta$ ipsa igitur est numerus pueri plenus scil.

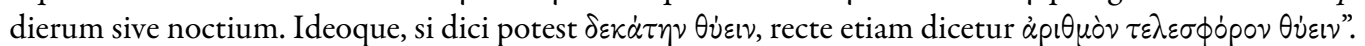

12 La congettura di Elmsley (1813, p. 93, ad Eur. Hcld. 603) è quasi certamente giusta (scambio in maiscuola fra $\Delta$ e $\Lambda$ ), nonostante la prudenza di Denniston (1939) e di Basta Donzelli (1995 ad l). In ogni caso, anche mantenendo $\lambda \dot{\varepsilon} \gamma$ ', l'espressione generica ('dille i giorni necessari perché una puerpera ritorni pura') può essere intesa come corrispondente al numero dieci.

13 La corruzione che interessa la prima parte del verso non ne cambia il senso generale. Per una panoramica delle proposte di correzione e interpretazione cf. Distilo (2012, pp. 314-15).

14 Cf. Kubo (1967, pp. 15-21 e 29). Kubo ritiene che qui Euripide riprenda un motivo frequente in miti di questo tipo (cf. la storia di Danae e Perseo, o il racconto erodoteo su Mandane madre di Ciro in Hdt. I 107 ss.)

15 Cf. in particolare i vv. 34-42.

16 È questa la più corretta lettura del passo proposta da Schwinge (1968, p. 133 n. 30); cf. anche Basta Donzelli (1978, p. 145 n. 21). Pericolosa potrebbe essere semmai la verginità di Elettra, che mantiene viva la possibilità di generare da un altro uomo un figlio nobile. Elettra non a caso ha tenuto nascosto il fatto a Egisto, cf. vv. 270-71.

17 Cf. Denniston (1939 ad l.): "In any case, she will want to put herself right with public opinion, whether she is devoid of genuine sympathy or not". Denniston comunque inclina a ritenere che Clitemestra sia stata turbata dalla nascita di un maschio.

18 Basta Donzelli (1995) scrive al v. 662 oủkovv invece di oủkoṽv, perché la domanda "nasce da uno sdegnato stupore per le riserve da lui (scil. il Vecchio) avanzate" (Basta Donzelli, 1978, p. 148).

19 Su questo aspetto della scena cf. Medda (2007, pp. 62-63 = 2013, pp. 107-108).

20 Le considerazioni svolte nel testo aggiungono un argomento a quelli già apportati da più parti a favore della trasposizione

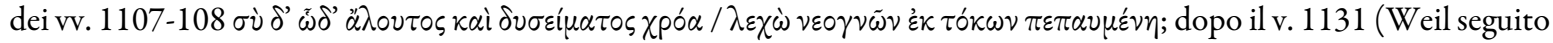
da Denniston, 1939, e dubitanter da Basta Donzelli, 1995). Con i due versi nella posizione in cui si trovano nel codice L, sembra impossibile che Elettra possa dire al v. 1124 'penso tu abbia sentito parlare del fatto che ho partorito'. Distilo (2012, pp. 550-51) ritiene che essi versi vadano sì rimossi dalla posizione tràdita, ma non collocati dove vorrebbe Weil, perché dopo il v. 1131 essi "appaiono decontestualizzati e slegati da ciò che segue e ciò che precede; anche in questa nuova sede, infatti, le parole della regina da un canto pongono fine alla discussione in modo brusco con l'introduzione di 
un argomento del tutto estemporaneo (avrebbe dovuto accorgersi prima delle condizioni della figlia), mentre dall'altro non danno ragione di quanto si affermerà a vv. 1132-1133”. Non credo che questo giudizio colga nel segno. La domanda relativa allo stato miserevole di Elettra si connette in realtà in modo efficace con la constatazione immediatamente precedente della mancanza di amici che consegue alla povertà, e contribuisce a far compiere a Clitemestra il passo finale verso la decisione di compiere il sacrificio. L'argomento relativo al fatto che la madre avrebbe dovuto accorgersi già da prima delle condizioni della figlia non ha valore: com'è normale nel dramma, il dato viene sottolineato nel momento in cui diviene funzionale (del resto, anche se i versi restassero dove si trovano in L, Clitemestra avrebbe comunque ignorato la cosa per più di cento versi dal momento del suo arrivo). Mastronarde (1979, pp. 91-2) difende la collocazione tràdita e trova che la domanda di Clitemestra, se posta prima di 1132, risulterebbe "sharp and unsympathetic", in contrasto con l'uso di $\sigma \grave{\nu} \delta$ ' $\bar{\omega} \delta \varepsilon$ che altrove introduce di regola una "apistetic question" con sfumatura simpatetica. Ma proprio di una domanda di questo tipo si tratta qui: Clitemestra cade nel tranello, facendosi prendere da un momento di debolezza di fronte alle condizioni della figlia e assume un atteggiamento simpatetico, accettando di farle un favore che le sembra innocuo.

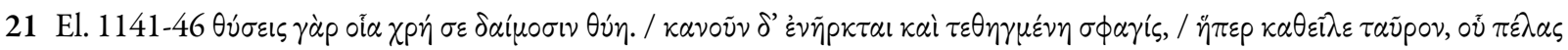

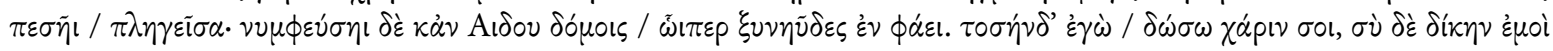
$\pi \alpha \tau p o ́ s$.

22 Il testo è citato dall'edizione di J Diggle (1981).

23 Cf. vv. 34-40, 73-76 (sacrificio umano), vv. 939-60 (Choes: Dunn, 1996, pp. 50-1), vv. 1040-1045 (più che probabile allusione ai Plynteria, su cui cf. Parker, 2005, p. 163), vv. 1096-1102 (Delia), vv. 1449-61 (Tauropolia), vv. 1462-70 (Brauronia). Sia consentito rinviare a Taddei (2009 e 2020, pp. 94-112).

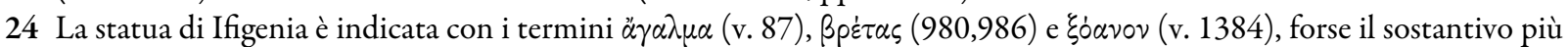
pertinente, se si considera la facilità con cui viene spostato. Sugli usi di quest'ultimo sostantivo nei tragici si veda Parker (2016, p. 329).

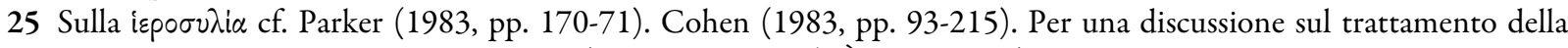

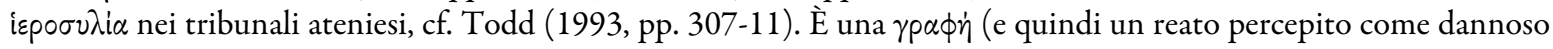

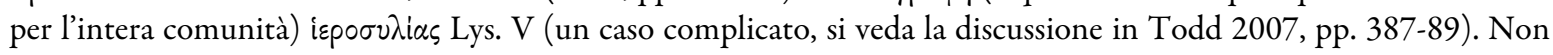
abbiamo molte notizie di processi di questo tipo, probabilmente perché chi veniva sorpreso a rubare oggetti sacri era

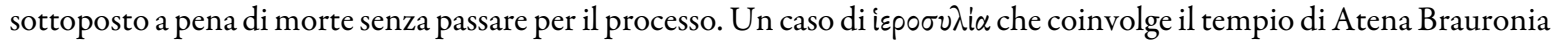
è in Din. II 12. Senofonte (Hell. I, VII 23) riferisce di una legge secondo la quale era proibito seppellire in Attica chi si fosse macchiato di questo reato.

26 La funzione di Ifigenia presso il tempio di Atena è designata tanto dal termine di sacerdotessa (įpéa, nella forma attica

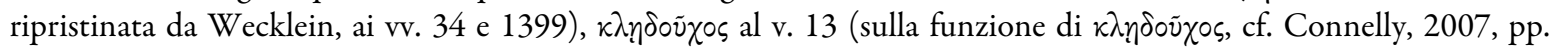

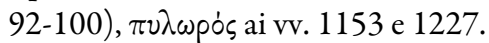

27 Atene e la terra dei Tauri sono frequentemente contrapposte nel corso del dramma (cf. vv. 1087 s., 1482 s., 1131, 1449), ma la comunità taurica non è descritta solo come isolata (cf. vv. 595, 1423-7). Sul tema si vedano le utili osservazioni di Saïd (1984 e 2002). L'opposizione tra Greci e Tauri non è, d'altra parte, monolitica e conosce momenti di vera e propria inversione come quando Toante (definito sovrano $\beta \dot{\alpha} p \beta \alpha \rho \circ \beta \beta \alpha \rho \beta \dot{\alpha} \rho \circ \sigma \sigma \mathrm{l}$ al v. 31) esprime sdegno nei confronti del matricidio commesso da Oreste, dicendo che nemmeno tra i Barbari una simile azione sarebbe stata concepibile (cf.

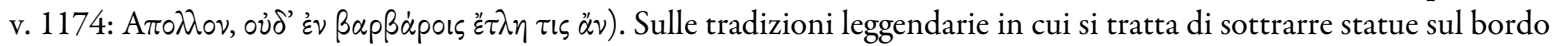
del mare si veda ora Ellinger, 2021 (con specifico approfondimento sulla Ifigenia tra i Tauri alle pp. 165-171).

28 I due sostantivi rinviano rispettivamente alla parte iniziale del sacrificio, vale a dire l'abluzione delle mani $(\chi \dot{\varepsilon} \rho \nu \imath)$ e alla raccolta del sangue delle vittime ( $\sigma \phi \alpha \gamma \varepsilon \tilde{\imath}$ ', che indica il contenitore per raccogliere il sangue, ed è correzione di Musgrave per l'ametrico $\sigma \phi \dot{\alpha} \gamma$ l', che invece avrebbe alluso all'intero rito di sgozzamento). Ai vv. 40-41 Ifigenia aveva precisato prendendo le distanze dal sacrificio umano del quale la dea si compiace (vv. 35-36)- di occuparsi solo dell'avvio del

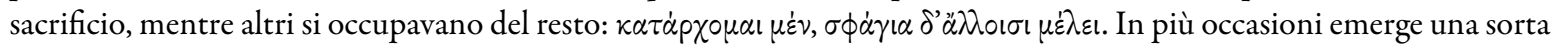
di impazienza dei Tauri (così Kyriakou 2007, p. 131) nella volontà di uccidere gli stranieri: cf. vv. 1153-54, 1190.

29 Il verbo viene usato due volte nella tragedia (qui e al v. 40), in entrambi i casi per indicare il compiacimento distorto che la dea prova nei confronti del sacrificio umano.

30 Nella caratterizzazione di Ifigenia come sacerdotessa scrupolosa, il testo riprende qui il verbo $\phi p o v \tau i \zeta \omega$ già usato al v. 343 , ancora da Ifigenia, anche in questo per riferirsi alla necessità di occuparsi della corretta amministrazione del sacro. In entrambi i casi l'uso del verbo è preceduto dall'esclamazione عĩદv ("at the beginning of a speech, dismissing the rapturous embraces and getting down to business”: cf. Stevens 1976, p. 34 con ulteriori esempi).

31 Ifigenia dichiara di essere arrivata ad elaborare un piano ( $\varepsilon \zeta \gamma \dot{\alpha} \rho \delta \dot{\eta} \tau \iota \nu^{\prime} \eta^{\prime} \kappa o \mu \varepsilon \nu \lambda \dot{o} \gamma \circ \nu$ ). Il nesso $\delta \dot{\eta} \tau \iota \nu \alpha$ (usato anche ai vv. 526 e 545) indica che la protagonista ha già chiaro ciò che intende fare, ma non fornisce all'uditorio i particolari. Cf. Denniston (1954, pp. 212-13).

32 Si vedano i vv. 735-92: il rituale che accompagna il giuramento è descritto con estremo dettaglio e prevede una ripartizione dei ruoli definita, con Oreste che svolge una funzione iniziale di guida (si osservino in particolare i suoi 


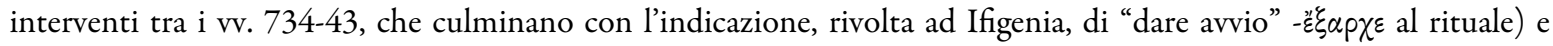
progressivamente si defila lasciando il rito nelle mani dei soli due contraenti, che lo articolano nella sticomitia dei vv. 743-54 in cui viene formulato un primo giuramento, e nel dialogo dei vv. 755-771, nel corso dei quali viene pronunciato un nuovo giuramento che tiene conto delle nuove condizioni). Sia consentito rinviare a Taddei (2021).

33 Molto dipende da quanto si possano presupporre conosciute le informazioni circa le pratiche tauriche di cui parla Erodoto (IV 103). Si vedano Hall (1989, pp. 111-12 e -sulla rappresentazione della crudeltà dei barbari- pp. 157-59).

34 Oreste e Pilade arrivano al tempio con le mani legate (v. 456) e identificati come le vittime più recenti destinate alla

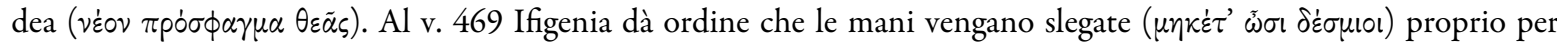
permettere lo svolgimento del sacrificio (cf. Plato, Crit. 119 d, Prot. 320a), e mantenute slegate anche quando Ifigenia si reca nel tempio per preparare la tavoletta necessaria per il giuramento (i $\pi p o ́ \sigma \pi 0 \lambda$ or -sulla cui identità molto si discute, cf. Kyriakou (2007, pp. 212-13)- devono sorvegliarli, ma $\delta \dot{\varepsilon} \sigma \mu \omega \nu \alpha \ddot{\tau} \tau \varepsilon$, v. 638). Il riferimento all'incatenamento diventa poi cruciale ai vv. 1202-205, quando Toante segue Ifigenia nella preparazione fittizia del sacrificio ai vv. 1202-1205 (Ifigenia sollecita gli schiavi a prendere le catene, sebbene Toante non veda possibilità di fuga). Il fatto che sia Ifigenia a tenere in mano i $\delta \dot{\varepsilon} \sigma \mu \alpha$ (v. 1333) è, poi, uno degli elementi che insospettiscono gli schiavi (che erano stati inviati proprio a prendere le catene: v. 1328) quando vedono la $\pi \circ \mu \pi \eta \dot{~ s i m u l a t a ~ c h e ~ p r e p a r a ~ i l ~ f i t t i z i o ~ r i t u a l e ~ d i ~}$ purificazione, ed è quindi uno dei segnali che l'inganno è riuscito. Cf. infra.

35 Sulla opportunità che gli animali siano "liberi" al momento del sacrificio, cf. Burkert (2003, pp. 147-50) con la discussione in Van Straten (1995, pp. 100-102). Più in generale, cf. Bremmer (2007).

36 In tragedia il verbo "non ha mai una corrispondenza scenica immediata, ma si riferisce ad atti da compiersi in un momento (talora anche in un luogo) non coincidente con quello in cui avviene il dialogo" (Medda, 2017, p. 447).

37 Sugli usi di $\theta \dot{v} \omega$ si vedano le osservazioni di Parker (2011, pp. 124-70). Una messa a punto sull'argomento si può leggere in Mugelli (2019), con bibliografia ulteriore.

38 Sui ruoli femminili nell'azione sacrificale Osborne (1993, pp. 400-401) e Parker (2005, p 276, n. 27).

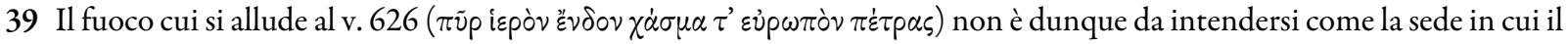
corpo viene bruciato (come accade invece a Cartagine, secondo la testimonianza di Diod. Sic. XX 14, 6-7), ma del fuoco custodito in modo perpetuo all'interno del tempio, con il quale verranno accese le fiaccole, utili in seguito per appiccare il fuoco alla pira con cui i cadaveri vengono cremati. Diversa l'opinione di Platnauer e Cropp, sulla base di Corbett (1970, pp. 150-52). Si veda la discussione in Parker (2016, p. 188).

$40 \pi \varepsilon i \sigma \omega \sigma \phi \varepsilon$, afferma Ifigenia al v. 743. Torneremo più avanti tanto sul tema della persuasione (cruciale anche nell'Elena) quanto sul rapporto tra spazio interno al tempio ed esterno ad esso. Sulla corretta interpretazione dell'espressione $\dot{\varepsilon} \nu$ $\pi \alpha p \alpha \sigma \tau \dot{\alpha} \sigma \iota \nu$ (v. 1159), cf. Di Benedetto e Medda (1997, pp. 139-40).

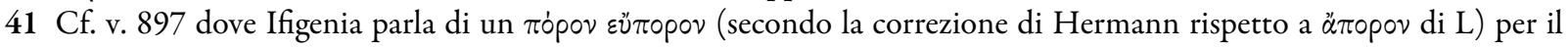
fratello, nei confronti del quale sente tutta la responsabilità della creazione di una via di fuga (cf. vv. 882-84).

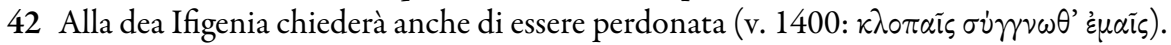

43 Cf. anche il v. 1215 dove Ifigenia ribadisce a Toante l'indicazione di restare $\pi$ pò $v \alpha \omega \tilde{\nu}$.

44 Il verbo è ripreso anche al v. 1156, quando il Coro risponde a Toante, che è appena entrato in scena e vuole sapere se il sacrificio è già stato celebrato, che sarà la stessa Ifigenia a dirlo con chiarezza (v. 1156: épeĩ $\sigma \alpha \phi \tilde{\omega} \varsigma)$

45 Nell'arco di pochi versi la statua viene indicata con due diversi sostantivi: v. 1038: پ’ $\gamma \alpha \lambda \mu \alpha$; v. 1040, 1044: $\beta p \dot{\varepsilon} \tau \alpha \varsigma$.

46 Sull'uso dell'acqua di mare come elemento utile a purificare si vedano le utili osservazioni di Beaulieu (2019, in particolare le pp. 207-208 sull'Ifigenia).

47 Cf., rispettivamente, Parker (1983, pp. 226-230 e 2005, p. 378 e 478). Sui Plynteria si veda anche Larson (2007, pp. 45-8).

48 Il tema dell'agire di nascosto era già emerso ai vv. 995-96 e 1024.

49 Mi riferisco in particolare alla costruzione di $\phi i \lambda i \alpha$ con il coro (anch'essa qui cercata attraverso la persuasione: cf. v.1053,

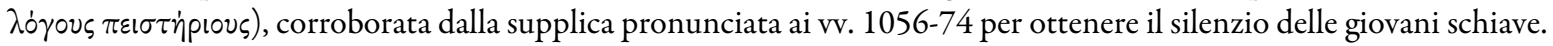

50 Nell'arco del dramma Ifigenia rivolge tre preghiere alla 'sua' dea: oltre a quella qui pronunciata, si vedano le preghieri presenti ai vv. 1230-33 e ai vv. 1398-402.

51 Si pensi al ruolo nello svolgimento del rituale del giuramento (vv. 735-80, cfr. supra) o all'indicazione data ad Oreste (v.

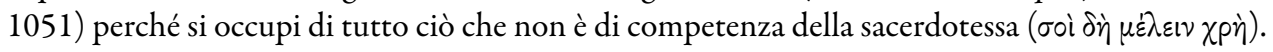

52 Cf. vv. 1180, 1202, 1212, 1214.

53 L'ipotesi è stata avanzata da F. Meinel (2015, pp. 140-71).

54 La preparazione e la cura di vesti per le statue degli dei erano parte ordinaria dei culti ateniesi (e non solo: si pensi al caso degli Heraia argivi, cf. Larson 2007, pp. 30-31). Si consideri, per esempio, il peplo che veniva tessuto dalle arrèfore e poi offerto ad Atena in occasione delle Panatenee. Cf. Barber (1992) e Parker (2005, pp. 163 e 269, n.71).

55 Cf. per esempio Soph. Ant. 1146, Eur. Ion 1076, Eur. Bacch.725. Il legame è serrato anche nell'iconografia. Come afferma R. Parker (2005, p. 350), "Eleusinian iconography depicts, one might almost say, torches, torches, nothing but torches". 
56 Sull'uso del fuoco (unito spesso a zolfo) per purificare cf. Parker (1983, pp. 227-28). È proprio R. Parker (1983, p. 228 , nota 18), tuttavia, a ricordare che la documentazione epigrafica fa riferimento anche a casi in cui è l'oro ad essere utilizzato per purificare. Una messa a punto su $\mu i \alpha \sigma \mu \alpha$ e purificazione si può leggere in Parker (2018).

57 Cf. Aristot. Ath. LVII 4 (દ่v iં tetto). Si vedano, in proposito, Mac Dowell (1963, pp. 39-40) e Boegehold (1995, pp. 43-44). Il contatto con la dimensione giudiziaria trova riflesso nel lessico utilizzato ai vv. 1178-80: alla scoperta dello stato di contaminazione (v.

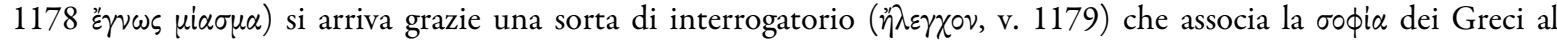
prodigio della statua che si muove. Il verbo $\dot{\varepsilon} \lambda \dot{\varepsilon} \gamma \chi \varepsilon\llcorner\nu$ appartiene al linguaggio giuridico (cf. Antipho I 12 e Aesch. $A g$. 1351, con le osservazioni di Medda, 2017, p. 302).

58 Così ai vv. 1164, 1166, 1178, 1196.

59 Il testo dell'Elena è citato secondo l'edizione di J. Diggle (1994)

60 Si vedano Platnauer (1938, p. XV) e la discussione in Marshall (2015, pp. 45-9, con ulteriore bibliografia).

61 Cf. Eur. IT vv. 335, 623, 864 (si veda supra).

62 Cf. Eur. Phoe. 972, dove è Creonte a usare queste parole rivolgendosi a Meneceo. Il tema dell'opportunità di allontanarsi in fuga 'da quella terra' -così cruciale per l'intero dramma- torna a più riprese: Elena suggerisce infatti in più occasioni la fuga da quella terra al suo interlocutore: oltre al verso qui esaminato si vedano i vv. $152(\gamma \tilde{\eta} \nu \tau \dot{\eta} \nu \delta \varepsilon \phi \varepsilon \tilde{v} \gamma \varepsilon), 805$ ( $\phi \varepsilon \tilde{v} \gamma \varepsilon \delta$ ’

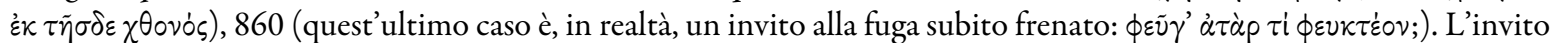
alla fuga si accompagna anche ad una sollecitazione a distaccarsi dall'etica eroica: Cf. vv. 806-808, dove Menelao descrive l'idea della fuga come indegna del valore guerriero di chi ha combattuto a Troia.

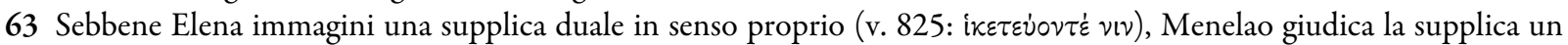

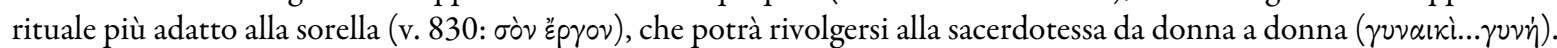

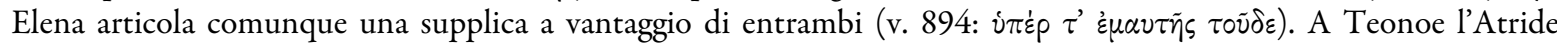
rivolgerà un lungo discorso (vv. 947-95), esplicitamente differenziato dai gesti della supplica (cf. infra, n. 65) ritenuti indegni di un guerriero (vv. 948-49). Anche Adrasto nelle Supplici euripidee esprime vergogna al momento di

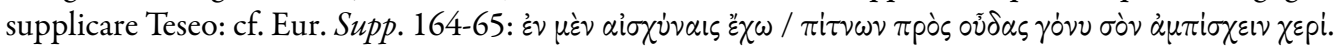

64 Per suggellare il patto di fuga che stanno stringendo Elena e Menelao articolano un breve rituale di giuramento (vv. 835-40), un ulteriore elemento di contatto con l'Ifigenia (cf. supra, n. 32). Il giuramento, che riprende quello già formulato da Elena ai vv. 353-6, è accompagnato da una maledizione invocata sulla testa di Menelao (si veda in proposito Torrance, 2009, pp. 2-3) e realizzando un contatto utile a rendere definitivo il vincolo (in questo caso devono toccarsi le due mani destre: v. 838). Si tratta di un giuramento che -come ha rilevato J. Fletcher- non ha effetti sullo svolgimento del dramma (Fletcher, 2012, p. 218). Un'importante e lucida analisi di questo giuramento è in Torrance (2009). Più in generale, sui giuramenti femminili in Euripide si può leggere Fletcher (2003).

65 Nel rituale di supplica giocano un ruolo importante la gestualità (il fatto di gettarsi ai piedi della persona supplicata, v. 894 per esempio) e anche il riferimento alla tomba (v. 961: $\dot{\alpha} \mu \phi i \mu \nu \tilde{\eta} \mu \alpha$; v. 1009: $\dot{\alpha} \mu \phi i ~ \tau \dot{\jmath} \mu \beta \omega)$. Sul rituale di supplica, cf. Giordano, 1999 e Naiden, 2006.

66 Mantengo qui la lezione di L, senza quindi accettare la correzione di Jackson accolta da Diggle.

67 La disticomitia in cui sono coinvolti Elena e Menelao nei vv. 1032-1084 contiene alcuni elementi di regolarità, come la corrispondenza dei tre ottativi che aprono gli interventi dei due interlocutori ai vv. 1039-44 (1039: $\pi \varepsilon ı \sigma \varepsilon i \alpha \varsigma ; 1041$ :

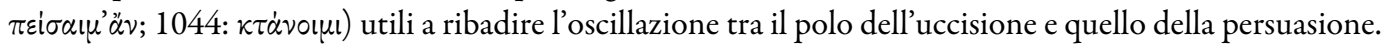

68 Il naufragio della nave di Menelao è stato descritto ai vv. 408-13. A differenza di Menelao, che si accorge ora della mancanza di un'imbarcazione su cui fuggire, Elena dà per acquisita la presenza di una nave al v. 1062.

69 Cf. vv. 1459-65.

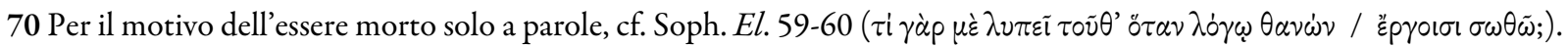

71 Nella gestione del rito funerario esisteva un vero e proprio 'codice cromatico' (Frisone, 2000, p. 66) che individua nel

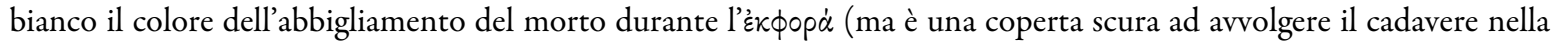
iscrizione della fratria dei Labiadi di Delfi e una veste color porpora poteva essere usata a Sparta: Frisone, 2000, p. 67). Sono invece nere le vesti indossate da chi partecipa al rito funerario (cf. Eur. Alc. 216, 427). E nero è il colore delle vesti di chi partecipa al rito funerario (Parker, 1983, p. 35). Sui culti funerari in Grecia antica si veda Sourvinou-Inwood (1996); sulle lamentazioni funebri cf. Palmisciano (2017).

72 L'espressione (già archilochea: fr. 213 W2.) torna identica al v. 1436, ancora connessa con il rito che Menelao dovrebbe celebrare in mare. Cf. infra.

73 La situazione è analoga a quella, già osservata, che si crea al v. 1176 della IT, quando Toante anticipa le spiegazioni che

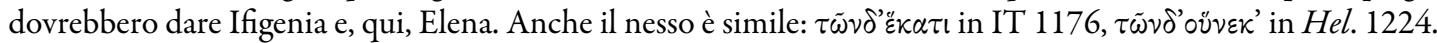

74 Sull'uso della particella colloquale $\delta \alpha i$, cf. Denniston (1954, 262-64) e le osservazioni di Kannicht (1969, pp. 320-21).

75 Il tema della lamentazione per i morti in mare ha una sua tradizione che va da Archiloco (fr. 13 W2.) almeno fino agli epigrammi funerari callimachei. Si vedano le osservazioni di Palmisciano (2017, pp. 137-44, su Archiloco, e pp. 363-65, 
Callimaco). Quanto al rito funerario svolto si fa in genere riferimento a cenotafi (si veda, del resto, il v. 1060), non a rituali in mezzo al mare, come quello qui rappresentato.

76 Gli animali destinati al sacrificio dovevano corrispondere a caratteristiche precise (tra queste il fatto di essere senza difetti: cf. Bremmer (2007, pp. 133-36), Lupu (2005, pp. 54-56).

77 A rendere ancora più strana la simulazione del rituale sta il valore purificante tradizionalmente attribuito al mare da parte dei Greci, su cui si veda Parker (1983, pp. 210, 229-30). Sull'uso dell'acqua come elemento purificatore nella religione greca si veda Bendlin (2008). Cf. Eur. IT 1153. In Il. I 314 il mare è il luogo dove vengono gettate le impurità $(\lambda \dot{u} \mu \alpha \tau \alpha)$ che derivano dal bagno purificatore ( $\dot{\alpha} \pi \partial \lambda \nu \mu \alpha i v \omega$ è il verbo usato) cui si sottopongono i guerrieri, bagnandosi con acqua di mare (corretta l'interpretazione di Cerri 1996, pp. 140-41 contra Kirk e Burkert, che pensano invece ad una purificazione avvenuta a terra).

78 Si tratta, in questo caso, della casa nella quale Menelao è invitato ad entrare al v. 1296 ( $\varepsilon \sigma \varepsilon \lambda \theta \varepsilon$ ), e nella quale Elena è

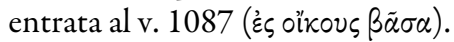

79 Si registra qui una lieve differenza rispetto a quel che accade nella Ifigenia, dove il coro assicura alla protagonista la propria fedeltà e il proprio silenzio (un elemento assai diffuso in tragedia), anche in quel caso ottenendo promessa di salvezza

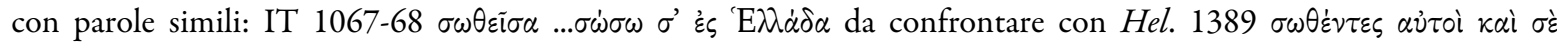
$\sigma \nu \sigma \sigma \tilde{\omega} \sigma \alpha i \pi 0 \tau \varepsilon)$. Sarà Afrodite, negli ultimi versi della Ifigenia, a ordinare a Toante di lasciare fuggire anche le giovani del coro. Nell'Elena non pare invece esserci alcuna prospettiva di fuga per il coro.

80 Il termine $\dot{\varepsilon} v \tau \dot{\alpha} \phi ı$ non è molto usato in età classica. In tragedia si trova, oltre che in questo passo, solo nell'Elettra di Sofocle (v. 326) ad indicare le offerte che Crisotemide sta portando con le proprie mani.

81 Il dato è tanto più interessante se si considera che Elena associa questa formulazione alla promessa di essere per Teoclimeno una donna che in casa si comporta come lui desidera (vv. 1407-408), e quindi rispettosa del sacro come il sovrano si era augurato al v. 1278. Diversa l'interpretazione di Allan, che vede in questa formulazione un'affermazione ironica ("i.e. no wife at all”, Allan, 2008, p. 313).

$82 \mathrm{La}$ forma ővaı entrava in quella circostanza in rapporto con l'espressione usata da Elena al verso immediatamente

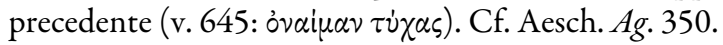

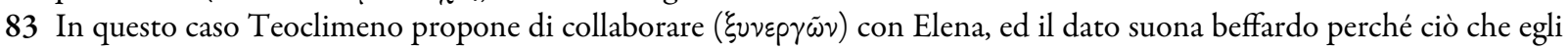
sta facendo è proprio collaborare con la sua interlocutrice, ma a tessere il proprio inganno.

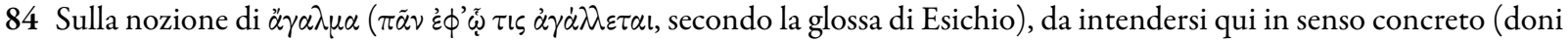
di nozze), cf. Eur. Tro. 1220, El. 871, con le osservazioni di Kannicht, 1969, p. 370.

85 Sull'uso del lessico e la sollecitazione delle competenze marinaresche in questa tragedia sia consentito rinviare a quanto ho scritto in Taddei (2020, pp. 151-54).

86 La situazione è analoga a quella che abbiamo osservato per l'Ifigenia, dove si crea un'opposizione analoga tra il sospetto

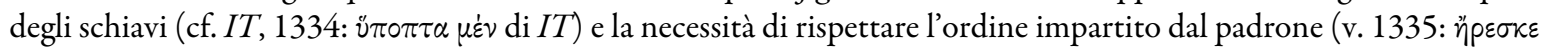

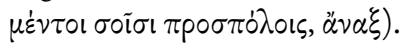

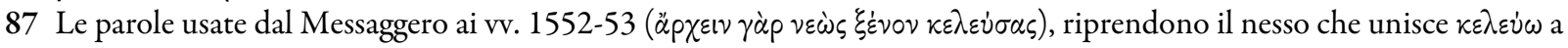
äpxદıv su cui Elena aveva giocato, come abbiamo visto, ai v. 1415-16.

88 A differenza di Diggle mantengo qui l'attribuzione dei versi al coro, e non al Therapon proposta da Clark. 